\title{
27. VARIABLE STARS (ÉTOILES VARIABLES)
}

PRESIDENT: M. W. Feast.

VICE-PRESIDENT: W. Wenzel.

ORGANIZING COMMITTEE: N. Baker, J. D. Fernie, H. Gursky, B. V. Kukarkin, L. Rosino, J. Smak.

SECRETARY: W. S. Fitch.

DIRECTOR OF INFORMATION BULLETIN: B. Szeidl.

\section{INTRODUCTION}

This report has been written by the President and by others whose names appear at the heads of the sections for which they are responsible. References are given by $A A A$ numbers or in an abbreviated form (see end of this report).

During the period under review the commission sponsored three meetings:

$I A U$ Symposium No. 59, 'Stellar Instability and Evolution' Canberra 16 - 18 Aug. 1973; 1974;

$I A U$ Symposium No. 67, 'Variable Stars and Stellar Evolution', Moscow 29 July-4 Aug.

$I A U$ Colloquium No. 29, 'Multiply Periodic Variable Stars', Budapest 1-5 September 1975.

The 'Information Bulletin' of the commission continues to be an important medium for rapid publication of short notes on variables of current interest. We regret the death of Professor L. Detre who for many years edited the bulletin. Fortunately the bulletin continues to operate from the Konkoly Observatory under the new editor Professor B. Szeidl. Professor B. V. Kukarkin and his colleagues of the Moscow variable star bureau continue their indispensable cataloguing work. They published the second supplement to the third edition of the General Catalogue of Variable Stars, the special supplement containing 1950 co-ordinates for the variables in the third edition of the catalogue and the 59th name list (IB 834). Prof. H. Sawyer Hogg published the third edition of her important catalogue of variable stars in globular clusters (Publ. David Dunlap Obs. 3, No. 6). Dr W. Wenzel writes that the Sonneberg Observatory, the card catalogue of the bibliography of variable stars is being transformed to punched cards in order that it may be included in the data file of the Strasbourg Centre de Données Stellaires (Strasbourg Bull. No. 4). An important review of 'Pulsating Stars' which is particularly strong on the theoretical side was published by J. P. Cox (Rep. Prog. Phys. 37, 356).

\section{RED VARIABLES}

Some aspects of work on red variables in the old disc and halo populations have been reviewed by Feast (IAU Symp. 59) and Lloyd Evans (IAU Symp. 67). Amongst many photometric programmes on Mira variables some of the most extensive are; Eggen UBVRI (ApJ Sup 276, ApJ 195, 661, PASP 85, 42); Barnes (ApJ Sup 221), VRI of 239 Miras; Lockwood et al ( $A$ pJ Sup 209) 5 colour, narrow band in near infrared of 292 Miras. Strecher (Minnesota Report 15) $3.5 \mu$ light curves of 35 Miras. Other work includes $20 \mu$ observations of late type variables (Simon, $A J$ 79, 1054) and infrared observations from outside the atmosphere (Maran et al, $B A A S$ 6, 312); $U B V$ of Mira (Koester, $A A$ Sup 13, 133; Celis, $A A$ 22, 9). Wing and Lockwood ( $A p J 184,873$ ) discussed the extreme Mira IK Tau. An extensive programme of JHKL photometry of Miras is under way at SAAO (Feast, Catchpole, Glass, Lloyd Evans). Much of this work is aimed at the determination of bolometric magnitudes and temperatures for Mira and other late type variables, (e.g. Dyck et al, $A p J$ 189, 89; Flowers, $A A$ 41,391). A suggestion $(A p J 195,661)$ that the absolute median visual magnitude was independent of 
period for Miras is not in accord with observations (Feast). Other discussions of absolute magnitudes are (Foy, Heck, and Mennessier, $A A$ 43, 175; J. O'cchieppo, IB 768; Kelly, $M N A S S A$ 33, 79). The absolute magnitudes of Miras should be considerably improved when Ianna's work on Leander McCormick third epoch plates is complete $(B A A S 7,139)$. New proper motion of red variables were also determined in a joint SAAO-RGO programme (Clube, Azlan, and Russo).

Azlan determined statistical parallaxes for semi-regular variables $(M N 165,337)$. Further discussion of Mira kinematics was given by Gotska (Sov. Astr. 17, No. 5). Lloyd Evans ( $M N$ 1975) published his study of Miras in the region of the galactric centre. Amongst other things he finds a marked period-luminosity relation.

Variable radio emission was detected from $\mathbf{R}$ Aqr (a Mira in nebulosity) by the Kingston group (Nat 247, 532; Nat PS 246, 111). SY For was found spectroscopically to have a companion similar to Mira Ceti B (Feast, Obs 95, 19). Moffet and Barnes could not confirm rapid changes that had been reported in the Mira UX Cyg (IB 739). UY CMa was classed as an SRd (Erleksora Byall Inst. Ast. Dushanbe No. 61,31). An investigation of the period variability of long period variables was made $(\operatorname{Per} Z v 18,485)$.

A major publication was the revised catalogue of spectra of Mira variables (Keenan, Garrison, and Deutsch, ApJ Sup 262). The earlier catalogue is extended by the addition of nearly 800 new spectrograms. Spectra of nine Miras at minimum are given by Wyckoff and Wehinger (PASP 84, 424). Buscombe and Simon measured emission line intensities in 32 southern Miras ( $A S S$ 27, 187). The physical conditions in the atmosphere of the high velocity Mira S Car were extensively studied by Shinkawa ( $A p J$ Sup 218). Amongst other things she deduced a general metal deficiency of a factor of 10 and an overabundance relative to iron of barium, lanthanum and europium. Catchpole and Feast found the Se variable RZ Sgr to have a large Li abundance as did the slightly variable S star TT9 in the eta Carinae complex. A survey of $188 \mathrm{C}$ type and $188 \mathrm{~S}$ type stars reveal no other cases of abnormally strong lithium lines. Lithium abundances for 7 SR variables of M type were derived by Boyarchuk (Izv. Krymsk. Astrofiz. Obs. 46, 47). Technetium is present in some but not all Miras of M type (BAAS 7, 234, PASP 85, 187). It was also noted in the $\mathrm{N}$ star UX Dra $\left(P A S P\right.$ 86, 78). An apparent enhancement of ${ }^{50} \mathrm{Ti}$ over less-abundant titanium isotopes was noted (PASP 84, 647). Lambert, Brooke and Barnes established upper limits to the intensities of the $\mathrm{H}_{2}$ quadrupole rotation-vibration fundamental band in several Miras.

Jacobsen and Wallerstein (PASP 87, 269) do not confirm the 26.7-year periodicity in the velocities of the forbidden lines in the Mira R Aqr. A correlation between the premaximum emission line radial velocities and the magnitude was found in $\mathrm{R}$ Cyg (Se variable) (Wojslaw, PASP 86, 932).

The pulsations of red variables especially with regard to the occurrence of multiple periodicities were reviewed by Wood (IAU Colloq. 29). He finds that Miras are first overtone pulsators $(M N 171,15 \mathrm{p})$. Koester $(A A 30,391)$ combined published radial velocities of emission lines and infrared photometry of o-Ceti to derive a radius and temperature assuming radial pulsations. On the other hand Shawl (PASP 86, 843) suggested that the oscillations of o-Ceti are nonspherical. Schwarzschild $(A P J 195,137)$ finds that the surfaces of cool stars may be covered by a few, very large, convective elements. He suggests that time variation in these few elements may account for irregular light variation in red stars. The idea that Mira stars are the precursors of planetary nebulae has been further developed by Kaller and Sparks ( $A p J$ 192, 477 but see also Finzi et al, $A A 30$ 325). The formation of grains in, and their expulsion from, the atmospheres of red variables has continued to be the subject of theoretical work (Woolf, IAU Symp 52, AL 15, 177; Salpeter, $A p J$ 193, 579 and 585; Wickramasinghe, $A S S$ 28, L25; Walker, $A L$ 16, 115). The total rate of mass loss from Miras and its heating effect on the interstellar medium were estimated (PASP 84, 639).

Lunar occulation diameters for three Miras have been determined; V Cnc (Se) (McGraw and Angel, $A J 79,485$ ), R Leo (Me) (Nather and Wild, $A J$ 78, 628); IRC + 10011 (Zappala et al, $A p J$ $192,109)$. In the latter case observations of the occultation in the infrared allowed both the diameter of the star and of the surrounding dust cloud to be determined. Further extensive studies of microwave emission from Mira variables have been made. Correlations of the $\mathrm{H}_{2} \mathrm{O}$ 
and $\mathrm{OH}$ emissions with the infrared flux have been established (Schwarz et al, ApJ 187, 491 Harvey et al, ApJ Sup 248). New OH sources (mostly type II OH/IR) have been detected and accurate positions determined (Winnberg et $a l, A L 13,125 ; A A 38,145$ ). The $(V-I)$ colours and the shapes of the light curves of Miras were found to depend on the $\mathrm{OH}$ characteristics (Fillet et al, $A L$ 14, 135, 17th Liège Symp 233; Bowers, $A A$ 39, 473) (See also $B A A S$ 5, 440, 6, 340). Very long baseline interferometric observations of five type II OH/IR sources indicate that the size of the masering component is greater than $20 . " 1$ (Reid and Muhleman, ApJ 196, L35). An important discovery was that the velocity separation of the two $\mathrm{OH}$ components in Miras is a function of the period (Dickinson and Chaisson, ApJ 181, L135, ApJ 199, 131. This is similar to the relation between period and the difference in the optical emission and absorption line velocities. No $\mathrm{OH} / \mathrm{IR}$ sources have been found in globular clusters (Knapp and Kerr, $A J$ 78, 458). Variable SiO microwave emission has been detected from Mira type and SR variables. (ApJ 191, L33, L37, L97, 195, 359, 199, L111). There have been further developments of the theory of masering in Mira stars with infrared excesses $(A A 40$, 233).

Forrest et al. (ApJ 195, 423) discussed the relation between the intrinsic polarization and infrared excesses of Mira variables. Other polarization work on red variables is reported by Serkowski (IAU Circular 2712) and Dombrovskij et al. (Trudy Ast. Obs. Leningrad 29, 45, 30, 89). Work in this field is in progress also in Belgrade.

Very long baseline interferometry of VY CMa and other OH/IR sources has lead to important advances of our understanding of the detailed structure of these sources. (Moran et al., ApJ 185,535 ; Harvey et al., $M N 169,545$ ). No linear or circular polarization was found in the $\mathrm{H}_{2} \mathrm{O}$ emission of VY CMa (Bologne et al., ApJ 199, 86). An accurate $\mathrm{H}_{2} \mathrm{O}$ position for the variable was obtained $(A A 36,217)$. Interferometry at $5 \mu$ has resolved VY CMa and IRC 10216 and provided an upper limit to the size of NML Cyg (McCarthy and Low, BAAS 7, 410). Detailed studies of $\mathrm{CO}$ absorption at $5 \mu$ in VY CMa and NML Cyg provides further data on the structure of these objects. They also indicate ${ }^{12} \mathrm{C} /{ }^{13} \mathrm{C}$ ratio much less the 90 indicating that the objects are highly evolved (Geballe et al, BAAS 7, 464). Dust models of VY CMa were considered by Jura $(A J 80,227)$ and its distance further discussed by Humphreys (PASP 87, 433). For VY $\mathrm{CMa}$ and some other high luminosity red variables Derviz and Dombrovskij (Trudy Ast Obs. Leningrad 29,65) found no difference between the polarization in the continuum and in molecular bands. Long period variations of NML Cyg at $3.5 \mu$ were reported by Strecker $(A J 80,451)$ and a model for the object proposed (Herbig and Lorne, $A p J 189,73$ and 75 ).

Eggen has given $U B V R I$ observations for many small amplitude red variables of the disc and halo populations and discussed the $\mathrm{M}_{\mathrm{bol}}-(R-I)$ relation for these stars. He also discussed a class of small amplitude $\left(<0^{\mathrm{m}} \cdot 2\right)$ short period (20-40 day) red variables $(A p J 184,793)$. Radial velocities of a few small amplitude red variables were given by Lloyd Evans (Obs 94, 179). Lloyd Evans is studying variables in the NGC 371 region of the SMC. Lockwood and Zinter $(A J 78,47)$ identified 230 additional variables in the $2 \mu$ catalogue. Medvedev $(P e r Z v 18$, 449) measured energy distributions of six M type SR variables.

Microwave studies of the molecules in the envelope of the carbon star IRC +10216 have been carried out (Morris et al, ApJ 197, 603, 199, L47). Light variations of IRC +10216 and of other infrared carbon stars were found by Alksnis (AAA 11,113,030). Krempec (Act Ast) carried out spectrophotometric observations of carbon variables. She also investigated the relation of the infrared colours to the periods etc. (IB 977) and carried out photometry of variable carbon stars (Bul. Ast. Obs. Torun. No. 50/III) Keenan investigated spectral changes in SCe stars $(B A A S 5,39)$. Spectra of SC stars were studied quantitatively by Yamashita $(P A S J$ 25, 511) yielding massive overabundances of $\mathrm{Y}$ and $\mathrm{La}$ and $\mathrm{O} / \mathrm{C}=1 \pm 0.01$. Theoretical constraints on the evolution of $\mathrm{N}$ stars were discussed by Schlesinger (BAAS 4, 323).

Langer, Kraft, and Anderson (ApJ 189, 509) made the remarkable discovery that the abundance of the s-process elements in FG Sge were increasing with time. Several theoretical papers have followed this discovery (e.g. Sackmann et al, ApJ 187, 555; Ulrich et al, ApJ 192, 507) (See also Antipov, 18th Liège Symp. 477; Sparks Kutter, ApJ 183, L121).

The absolute magnitude of the lithium rich Se variable T Sgr was derived from its companion (Culver and Ianna, $A p J$ 195, L37). The bolometric absolute magnitudes of $S$ type variables 
was derived (Feast, Catchpole, and Glass, $M N$ ) from infrared observations and shown to be compatible with the operation of 'hot-bottom' convective theory (cf. Scalo et al, ApJ 196, 805).

The companion to W Aql (S3,9e) was discussed (Culver et al, BAAS 6, 427). Eggen gave $U B V R I$ photometry of $\mathrm{S}$ type variables and related objects and discussed their classification $(A p J 177,489)$.

\section{R CORONAE BOREALIS VARIABLES}

The RCB stars have continued to attract considerable attention. One reason for this is that they are in an advanced and little understood stage of stellar evolution in which they have lost their hydrogen rich atmosphere. Also they have, according to one view, the ability to form and eject circumstellar dust clouds giving large infrared excesses. On another view these excesses are due to an infrared companion. A recent general review of RCB stars has been given by Feast (IAU Symp. 67) and it seems unnecessary to repeat the discussion and references given there. Several RCB stars have been active during this period (brief report appearing in IAU Circulars and Telegrams). UBV photometry has been published for several stars (e.g. RY Sgr, $\mathrm{RCrB}, \mathrm{XX}$ Cam, Landolt, $P A S P$ 85, 625, 606; RCrB, Epps, $R O B 176$ etc). Andrews (Pretoria) monitored various RCB stars in $U B V$ and is at present analysing the data. $U B V$ observations of $\mathrm{R} \mathrm{Cr} \mathrm{B}$ listed by Nakagiri $(P A S J 27,379)$ for the 1973-74 minimum show in general greater absorption at a given $(B-V)$, (his Figure 2) than did the 1966-1970 observations of Fernie et al. (ApJ 172, 383). Pretoria work $(M N 158,305)$ on RY Sgr showed that chromospheric emission had this effect (by reducing the observed $(B-V)$ ). Evidently more work is needed to distinguish between changing chromospheric effects on the colours and real changes in the reddening law of the circumstellar shell.

Humphreys and Ney $(A p J 190,339)$ and Strecker $(A J 80,451)$ found a slow variation in the $3.5 \mu$ brightness of RCrB with an amplitude of about $1^{\mathrm{m}} .5$. They believe this may be periodic. Variations of about the same amplitude have been found in RY Sgr (SAAO infrared group). RY Sgr and S Aps have been monitored at SAAO in JHKL. For an understanding of the nature of RCB stars it is important to know whether all of them are like RY Sgr in being pulsating variables. Griffin observed $\mathrm{RCrB}$ intensively with his radial velocity meter at Cambridge in April - August 1975. The result shows a rather definite periodicity of about 2 months, presumably due to pulsations. It has been inferred on spectroscopic and infrared grounds that dust is ejected asymmetrically from RCB stars (see summary Feast, $I A U$ Symp. 67). Wdowiak ( $A p J$ $198,439)$ has suggested that the surfaces of RCB stars are covered by a few large convective cells. Dust formed in one or more of these will be ejected by radiation pressure giving rise to the RCB phenomenon. Maron (Nat 252,214) has suggested that the initial drop in light of RCB stars may be due to the formation of Platt particles. Krebwski (IAU Symp. 67) appears to have duplicated in the laboratory the violet continuum found in RY Sgr during minimum. $U B V$ observations of southern RCB stars have been made by Sherwood (IAU Symp. 67). Totochava finds evidence of short period $(\sim 2 \mathrm{~h})$ variations in XX Cam and Shulman discusses the dust shell theoretically $(I A U S y m p .67)$. Landolt $(A p J 196,789)$ finds the helium stars HD 160641 and $+13^{\circ} 3224$ to be variable. The latter with a period of 0 d 1 .

\section{RV TAURI STARS}

The absolute magnitudes of the RV Tau stars have been discussed by Du Puy ( $A p J 185$, 597) and later by Barnes and Du Puy ( $A p J$ 200, 364). Initially large amounts of neutral extinction around RV Tau stars were postulated. However, the second paper shows that there is no evidence for this.

Infrared excess due to circumstellar dust is present in most RV Tau stars (Gehrz and Ney, $A p J 178,715 ; P A S P 84,768)$. It was initially supposed that the presence or absence of the 'silicate' emission bump and other features in the infrared would allow the carbon-rich and 
oxygen-rich RV Tau stars to be distinguished from infrared observations alone. However Lloyd Evans $(M N 167,17 \mathrm{p}$.) has shown that the situation is considerably more complicated. Further work to unravel these complications is desirable.

Photometry of $\mathrm{U}$ Mon and $\mathbf{R}$ Sct including an unusually deep minimum of the latter was published by Wisse and Wisse $(A A 23,463)$. U Mon and R Sct show He I $10830 \AA$ in emission probably due to shock waves and $\mathrm{R}$ Sct in addition shows infrared emission of $\mathrm{Ti} I$ and $\mathrm{Si} I$ lines (Zirin, BAAS 7, 470). Several RV Tau stars including $\mathrm{U}$ Mon and $\mathrm{R}$ Sct are being monitored photometrically in the infrared and visual regions and also spectroscopically at SAAO (Cape). A rather extensive survey of RV Tau stars at $1612 \mathrm{MHZ}$ failed to detect any $\mathrm{OH}$ emission from these stars (Bowers and Cornett, $A L$ 15, 181). Lloyd Evans (SAAO) has carried out a large programme of low dispersion spectroscopy of RV Tau stars as part of a more general survey of type II cepheids (cf $I A U$ Symp. 67, 555). The curious variable V725 Sgr with a changing period may belong to the RV Tau class (Demers, JRASC 67, 19).

\section{SYMBIOTIC STARS AND RELATED PECULIAR OBJECTS}

A general review of symbiotic stars by Boyarchuk and a number of contributed papers appear in IAU Symp. 67. Probable symbiotic objects were identified in the Magellanic Clouds (Feast, Webster, $M N 168,31 \mathrm{p}$ ). Infrared work on symbiotics shows the presence of cool stellar components and dust emission (e.g. Glass and Webster, $M N$ 165, 77; Woolf, $A p J$ 185, 229; Webster and Allen $M N 171,171$ ). Low resolution scanning of symbiotics has been carried out by A. Walker (Cape). A number of symbiotics are being monitored in the infrared for variability (Cape). The light curve of HBV $475=\mathrm{V} 1329 \mathrm{Cyg}$, a radio emitting symbiotic, has been derived from 1950 to 1973 (Hicks, IB 804; Arhipova and Mandel, IB 762; IAU Symp. 67). The wavelength dependence of the (high) polarization in MWC 349 was found to be like that of VY CMa (Elvius, $A A$ 34, 371). Olnon $(A A 39,217)$ discussed in detail the interpretation of the radio emission from the object as due to free-free emission. The peculiar binary $R Y$ Sct was detected at radio frequencies (Hughes and Woodsworth, Nat PS 242, 116). Several general discussions of radio emission from symbiotics and related objects were published (Ciatti et al., $A A$ 38, 438, 39, 495; Panagia and Felli, $A A$ 39, 1; Oster, Unsold Birthday 1975 volume). The symbiotic radio emitter V $1016 \mathrm{Cyg}$ has been extensively studied spectroscopically (Ahern, $A p J$ 197, 639; FitzGerald and Pilavaki, $A p J$ Sup 258, Mammano and Ciatti, $A A 39,405$ ) and the relation to slow novae like RR Tel discussed. Infrared photometry (Harvey, $A p J 188,95$ ) indicates a period of $\sim 450$ days for the red component. Microwave $\mathrm{OH}$ emission may be present in V $1016 \mathrm{Cyg}$. It was not found in five other symbiotics (Lepine and Rieu, $A A$ 36, 469). The variability of the $Z$ And system is believed to lie mainly in the late type component (Lukatskaya, $\operatorname{Per} Z v 19$ 253). Variable polarization has been reported for $Z$ And (and also for HR Del and Z Cam) (Belokou and Shalov, Trudy Ast. Obs. Leningrad 30, 103). A study of the spectrum of the symbiotic star $\mathrm{CH}$ Cygni in the period 1961-1973 does not support the hypotheses that this is a binary system (Dentsch et al., PASP 86, 233). Colorimetry of the star has been published (Pub. Tartu Ast. Obs. 40, 163). Outbursts of CI Cyg have been reported (IAU Circ. 2788; IB 863; BAAS 5, 17). The orbit and spectroscopic characteristics of the eclipsing symbiotic AR Pav were derived by Thackeray and Hutchings $(M N 167,319)$ and a model proposed. Andrews ( $M N 167,635)$ gave photometry and a discussion of the eclipses of AR Pav. V 748 Cen (van Genderen, Glass and Feast, $M N 167,283$ ) may be a somewhat similar system but with total eclipses. The infrared excess of XX Oph (Bep) is due to a cool companion (Lockwood, Dyck and Ridgway, $A p J 195,385$ ). UBV of 12 symbiotics can best be explained on the binary hypotheses (Belyakina, Izv. Krymsk. Ast. Obs. 50, 103). Spectroscopic observations of $\mathrm{BQ}[]$ stars have been discussed (Ciatti et al., $A A$ 34, 181). Models of asymptotic giant branch stars have been constructed which have some similarity to symbiotic stars (Wood, ApJ 190, 609). The $34 \mu$ flux of $\eta$ Car is due to a dust shell (Becklin et al., ApJ 190, L69). Feinstein, Marroco $(A A 30,271)$ found a possible 3-year cycle in the brightness of $\eta$ Car.

The spectrum of the slow nova RR Tel and its changes 1961-1972 were discussed (Aller et $a l ., A S S$ 20, 93, 30, 387) and [Ca VII] emission lines identified (Thackeray $M N$ 167, 87). Fe II 
and [Fe II] intensities were used to support the idea of continuous ejection (Friedjung, ASS 29, L5). The evolution of the WR features in RR Tel was discussed (Thackeray and Webster, $M N$ $168,101)$. The infrared excess of $R R$ Tel found by Glass and Webster $(M N 165$, 77) was interpreted by Feast and Glass $(M N 167,81)$ as due to a cool star plus a dust shell. This is consistent with the presence of TiO bands (Webster, IAU Symp. 59). A cool component was also detected in the slow nova RT Ser. The object Henize 2-177 which resembles RR Tel has been extensively studied (Webster, $M N$ 164, 381; Carlson and Henize, $A p J$ 188, L47; Liller, $A p J$ 192, L89). A very slow nova (Henize 3-558) has been found (Henize and Liller, ApJ 200, 694). Hermanec discussed the evolutionary status of T CrB (IAU Symp. 66).

\section{BETA CANIS MAJORIS VARIABLES}

A review of observational aspects of multiperiodicity in $\beta$ CMa stars was given by Lesh and Aizenman (IAU Colloq. 29). About $50 \%$ of $\beta$ CMa stars are singly periodic the others have two or more periods. They suggest that the origin of multiperiodicity is intimately connected with the (unknown) basic instability mechanism. Deupree suggests that the beats are due to a degeneracy between a radial and a non-radial pulsation mode (ApJ 190, 631). Mahra and Sinvhal believe they have shown from studies of the interfering periods that $16 \mathrm{Lac}$ is in the early shell hydrogen-burning phase whilst $\gamma \mathrm{Peg}$ is in the core hydrogen burning phase. Extensive photometry of $15 \mathrm{CMa}, \xi^{\prime} \mathrm{CMa}$ and $\beta \mathrm{CMa}$ was discussed (Shobbrook, $M N 161,257,162$, 25). HR 6684 was placed in the $\beta$ CMa class (PASP 84, 718,85,632). Several new $\beta$ CMa stars were discovered by Balona. Simultaneous spectroscopy and photometry of $\sigma$ Sco indicates that the star is pulsating radially (Balona, Robertson). Variations of line profiles for $\beta$ Cep supports the radial pulsation hypothesis (Goldberg et al., AA 32, 355). The profiles of the hydrogen lines in $\beta$ Cep and $\nu$ Eri were interpreted as being affected by non-central emission (Laskarides, $A A$ 24,91 ). Dukes made a very extensive radial velocity study of Spica and detected a number of new frequencies $(A p J 192,81)$. Far ultraviolet observations of $\beta \mathrm{CMa}$ stars show that the amplitude increases with decreasing wavelength (Beeckmans et al., IAU Colloq. 29; Lesh, BAAS 7, 252).

Balona and Feast $(M N 172,191)$ deduced the luminosities of the $\beta \mathrm{CMa}$ variables from $\mathrm{H} \gamma$ equivalent widths. Their instability strip is parallel to the S-bend locus. It is $0 \div 3$ brighter than that given by Jones and Shobbrook $(M N 166,649)$. The instability strip and its significance for the evolution of $\beta \mathrm{CMa}$ stars was discussed earlier by Lesh and Aizenman $(A A$ 22, 229; IAU Colloq. 17). The instability strip and the range of masses of $\beta$ CMa stars was also discussed by Eggen ( $A p J 198,131)$. Balona and Feast also found $Q=0.027$ which agrees with the predictions for first overtone radial pulsations (Lesh and Aizenman, $A A$ 34, 203). Statistics of $\beta$ CMa stars were considered by Lesh and Aizenman $(A A 26,1)$ and by Percy $(A A 30,465)$ who concluded that the frequency of $\beta$ CMa stars was about $20 \%$. Shaw $(A A 41,367)$ gave $u v b y-$ $\mathrm{H} \beta$ photometry for $\beta$ CMa stars and also considered the statistics. He concluded that the $\beta \mathrm{CMa}$ stars are not solely in a secondary contraction or early shell burning stage of their evolution. The possibility that nuclear driven non-radial vibrational instability might be the basic cause of the $\beta$ CMa phenomenon was discussed by Lesh, Aizenman and $\operatorname{Cox}(A p J 197,399,195,175$, $I A U$ Colloq. 29). Cox and Tabor (IAU Symp. 59, 73) discuss the search for $\beta$ CMa type pulsation in extreme composition models. The theory of $\beta \mathrm{CMa}$ stars was also discussed by van der Borght and Jensen (PASA 2, 269) and by Chiosi $(A A 37,281)$. Osaki $(A p J 189,469)$ suggests that if the large scale convective motion is oscillatory, the frequency of one of the convective modes may coincide with the frequency of a non-radial oscillation of the whole star and this may excite non-radial oscillations. Varshavskij and Tutukov (Nauch Inf No. 20, 101) find $10<M / M_{\odot}<25$ for $\beta$ CMa stars. Shobbrook (IAU Symp. 59, 69) studied the position of $\beta$ CMa stars in the $\beta,\left[C_{1}\right]$ plane. Eggleton and Percy made a comparison between the observed period changes in $\beta$ CMa stars and theoretical values $(M N 161,421)$. Peters $(P A S P 85,536)$ finds the chemical composition of $\beta$ CMa stars to be similar to that of non-variable $B$ stars. 


\section{Am, AP, MAGNETIC VARIABLES}

A great deal of the work in this field is purely spectroscopic and this will be dealt with by Commission 29 (see also IAU Colloq. 32 'The Physics of Ap Stars'). A new catalogue of Ap and Am stars is in preparation (Catalano, Jaschek, Renson, Schoneich, and Weiss).

By monitoring Ap stars of tow $\mathrm{V}$ sin $\mathrm{i}$ in $u b v y$ Wolff and Morrison detected several new very long period Ap stars (PASP 85, 141). Renson and collaborators have also used Strömgren photometry to derive periods for Ap stars. The light and spectrum variations of HD 51418 (Ap) were studied by Gulliver and Winzer $(A p J 183,701)$. Kodaira $(A A 26,385)$ examined light curves of 49 periodic Ap stars and found they supported the variable blanketing model. Renson studied the radial velocities of different elements in $a^{2}$ CVn $(A S S 17,69)$. Adelman $(A p J 195$, 397) measured the continuous energy distribution of Ap stars.

HR 7129 was found to be an early type helium variable (like a Cen) (Balona and Martin, $M N$ $166,35 \mathrm{p}$ and in press). The star shows a variable magnetic field of large range (12 kG) (Wolff and Wolff, $P A S P$, in press). Babu and Sinvhal $(B A C 23,297)$ found that the mean rotational velocities of magnetic stars do not depend on their magnetic fields. Hesser, Walborn monitored the He variable $\sigma$ Ori E simultaneously spectroscopically and for continuum fluxes, $\mathrm{H} a$ profile and $u b v y \beta$ indices. No evidence for coherent activity has yet been found. Evidence for broad absorption features in a Cen was presented by Underhill et al. $(A p J 197,393)$ and a large magnetic field was found for the star (Wolff and Morrison, PASP 86, 935). Extensive photoelectric photometry of magnetic variables has been carried out at the Catania Observatory and various discussions published (e.g. $A J$ 77, 666, 78, 734; Blanco and Catalano, $A A$, in press; papers in IAU Colloq. 32). The need for international collaboration in the study of magnetic variables was stressed (IB 761). Megessier $(A A 39,263)$ applied the oblique rotator model to 103 Agr (Ap).

\section{MISCELLANEA}

Maximum entropy spectral analysis has been shown to be a powerful method of searching for periodicities (e.g. Richer and Ulrych, $A p J 192,719$ ).

Methods of period determination have been discussed by a number of workers (Astron. Astrofiz. Kiev. 19, 118; Per Zv 19, 117, Stud Cerc Ast 18, 37; Morraco and Muzzio (La Plata), unpubl.). The use of Fourier techniques to analyse periodicities of variables was extended by Gray, Derikachary $(A P J 181,523)$.

No evidence of short period $(2 \mathrm{~min}<P<1 \mathrm{~h}$ ) variations attributable to pulsational instability was found in WR stars. However some show variations which may be connected with their binary nature (Moffat and Haupt, $A A$ 32, 435). Night to night variations in the emission line strengths of WC stars were found (Bahng, MN 170, 610). Variations in C III 5696 in WC stars was also reported (Schumann, Seggewiss, IAU Symp. 67). Rablev reviewed WR stars (IAU Symp. 67). Night to night variability has also been established for CV Ser the 'Sometimes-eclipsing' WR star (Schild and Liller, $A p J$ 199, 432). No rapid spectral variations were found in 10 Lac (09 V) with an Image Isocon system (Glaspey and Walker, $P A S P$ 85, 188). Dean, White and Lockwood $(B A A S 6,427)$ observed a remarkable brightening $(\sim 0 \mathrm{~m} 4)$ of $\zeta$ Oph $(09.5 \mathrm{~V})$ in the near infrared although the star was not previously known as a variable. Simultaneous spectroscopic, polarimetric and photometric observations of the Be star $\pi$ Agr were carried out (Haefner et al., $A A$ 38, 203). A slow variation in the development of the shell was detected. Merlin ( $A A$ 39, 139) observed variations in $U B V$ of the Be star HD 174237. Feinstein (PASP, Aug 1975) studied light variations of Be stars and the 30-year periodicity in the shell of o And was confirmed (Fracassini, Pasinetti, IB 1044).

A review of 'S Doradus' variables was given by Sharov (IAU Symp. 67). Rosino and Bianchini discussed Hubble-Sandage variables in M 31 ( $A A$ 22, 453). Nevo et al. $(A A 42,247)$ obtained negative observations in a search for optical pulsations in spectroscopic binaries believed to contain black holes or neutron stars. Godoli reviewed stellar activity of the solar type at IAU Symp. 71. 


\section{VARIABLE STAR SURVEY WORK (W. Wenzel)}

\section{A. General Surveys}

At Bamberg Remeis Observatory the search for new variables on plates of several southern stations has been continued. Provisional and/or final results appeared in $I B$ and in Veroeffentl. Sternw. Bamberg X, the number of recorded Bamberg variables (BV) being now 1621 .

W. J. Miller of Fordham University Astronomical Laboratory, before his regrettable death, continued together with A. A. Wachmann the processing of newly discovered and of known variables of the Cep-Lac field (Ric. Astron. 8, No. 18 and 20), VV 428-490.

At the Kapteyn Laboratory of Groningen L. Plaut succeeded in re-determining the periods of the RR Lyrae stars of Baade's field near NGC 6522 (109 stars); up to then the period had to be considered suprisingly short as compared with the periods of similar stars elsewhere in our galaxy $(A A 26,317)$. Furthermore some supplementary results of the Palomar-Groningen variable star survey were given by Plaut in $A A$ Sup 12,35. An $U B V$ photoelectric investigation in the Groningen-Palomar fields was done by Wehinger and Hidajat at Kitt Peak to establish sequences for 170 stars covering the range of 6 to 8 visual magnitudes $(A J 78,401)$.

Deurinck and Goossens of the Astron. Institute of the Catholic University of Leuven reported newly discovered variables in several southern fields on plates taken with the 10 -in. Metcalf Telescope of Boyden Observatory (IB 792; 793; 794).

At Sonneberg Observatory some further progress has been achieved by H. Gessner, I. Meinunger, $C h$. Thänert, $H$. Busch and $H$. Haussler in processing the large number of faint variables discovered there. Detailed results were published concerning the fields of $\epsilon$ Pav (Veroeffentl. Sternw. Sonneberg 6, No. 5), a Pav (ibid.), $\beta$ Aps (6, No. 6), $\beta$ Her (8, No. 4), $\chi$ Lyr (ibid), $\gamma$ Phe (8, No. 5) and $\mu$ Ara I (ibid.); the fields of 20 Vir, $a$ Per and $\eta$ Ara II are being worked on. The Sonneberg Sky Patrol and the photography of special astrographic fields are continued and a large number of miscellaneous variable stars were observed on the plates of the file (see $M V S$ ).

G. Romano at the Observatory of Padova-Treviso has continued the survey of variables at different galactic latitudes of the Asiago program. The fields studied are $\gamma$ Com (Pubbl. Oss. Padova 164), $23^{\mathrm{h}} 17^{\mathrm{m}}+7^{\circ} 40^{\prime}$ in Peg (ibid. 166), the Cygnus cloud (ibid. 167) with a new electronic method of computation (G. Pinto), o And on the material taken from 1959 to 1974 , and (now in progress) $\beta$ Per plus a contiguous field.

\section{B. Surveys in Higher Galactic Latitudes}

van den Bergh and co-workers performed a search for faint variable objects in a region of altogether 6.2 square degrees near M 31 on Palomar Schmidt exposures. The aim was to detect active extra-galactic objects. 13 new variables were found $(A J 78,375)$, but L. Meinunger of Sonneberg Obse1 vatory questioned the extra-galactic nature of most of them $(M V S 6,90)$.

L. Meinunger continued the search for new variables off the Milky Way on plates of the Tautenburg 52-in. Schmidt telescope and the Sonneberg $40-\mathrm{cm}$ astrographs. Detailed results are published on the fields around M $3(M V S 6,37)$ and $\mathrm{M} 31(M V S 7,1)$. A hitherto unknown type of variability seems to have been detected by him in the case of 12 slowly varying uncoloured objects described in $A N 294,251$, one of them probably belonging to the globular cluster M 3. A summarizing note on five novae in the halo of M 31 is given in $A N 294,255$. An investigation of the surroundings of $\mathrm{M} 92$ will start soon, most of the plate material having been already secured at the Tautenburg and Sonneberg instruments.

\section{Surveys on Flare Stars and T Tauri Stars}

The search for new flare stars in clusters goes on in a number of observatories. The Pleiades are surveyed in a more or less combined program by the observatories of Asiago (Rosino and Pigatto, see for instance Mem. Soc. Astron. Ital. NS 44, 339; Contr. Asiago No. 273 and 296; $I B$ 776), Budapest (L. G. Balázs, Jankovics, Kun, and Szécsényi-Nagy, $I B \quad 803 ; 839 ; 898$ ), 
Bjurakan (Ambartsumyan and Parsamyan et al. for instance Astrofiz. 8, 485), Sonneberg (Gotz IB 771; MVS 6, 85) and Tonantzintla (Haro and Chavira, Bol. Tonantzintla 1, 3).

The confused situation concerning the seemingly high flare star activity in the Coalsack region appears now to be settled: The objective prism survey of the Warner and Swasey Observatory (Weaver, $A p J 184,881$ ) and the results of Mac Connell (van Vleck Observatory) and Dixon (Ann Arbor-Michigan) on Curtis Schmidt telescope multiple-exposure plates show $(A J 79,705)$, that the results of former investigations, which had yielded up to 140 rapidly varying stars in that region, must have been spurious. This is confirmed by considerations of A. D. Andrews (Armagh Observatory).

Weaver $(A p J)$ furthermore provides photographic UBVI magnitudes of 107 of the variables previously announced and concluded that they are reddened early type stars not associated with the Coalsack Cloud.

The results from investigating the association Cyg $\mathrm{T} 1$ at the observatory Hoher List of the University of Bonn were published in Veroeffentl. Bonn No. 87 by Gieseking. 32 variable stars and six $\mathrm{H} \alpha$-objects of apparently constant brightness, all belonging to the extremely young stars of that region, were photometrically studied.

At Uppsala Observatory Welin performed thorough searches and observations of $\mathrm{H} \alpha$-emission stars in and near NGC 7000; five papers on this subject underlie the summarizing work presented in Uppsala Report No. 4. This includes a discussion of the behaviour of V 1057 Cyg whose outburst was found during that survey. Some efforts were made in distinguishing between astrophysically different $\mathrm{H} \alpha$ objects of that region, above all between background Be-stars and the Orion population of the nebula. A sample of Welin's stars were also checked by Hudec et al. (MVS 6, 131,171; 7,63) with respect to variability, presence of $\mathrm{H} \alpha$ emission, and membership in the $\mathrm{T}$ association.

A number of further regions were surveyed to detect new Orion population members of associations, among them the Chamaeleon association (Henize and Mendoza, $A p J 180,115$; see also Feast and Glass, $M N 164,35 \mathrm{p}$, who investigated the nature of the $\mathrm{R}$ Mon type star No. 16), the Orion T associations (Brück, Publ. Roy. Obs. Edinburgh 7, 93; see also Andrews, Bol. Tonantzintla 6,161, who gave $U B V R$ data of 279 variable and $\mathrm{H \alpha}$ emission objects), and the group in the vicinity of RCrA (Knacke et al., ApJ 179, 847).

\section{VARIABLES OF THE ORION POPULATION} (W. Wenzel)

A lot of modern work on Orion population variables (that is, variables of extremely young evolutionary age) lays emphasis on circumstellar dust phenomena. Cohen performed and discussed infrared observations (four papers: $M N 161,85 ; 97 ; 105 ; 164,395$ ). In narrow bands from 2 to $12 \mu \mathrm{m}$ he observed samples of stars in the young clusters NGC 2264 and IC 5146, in the association Cyg VI, and in the grouping of H $\alpha$ emission objects of NGC 7000; furthermore a number of $T$ Tauri variables and early-type stars of the Orion population and early-type nebulous objects such as AB Aurigae have been investigated. In the proposed model mass infall and mass ejection play decisive parts in interpreting the observations. An infrared survey of $89 \mathrm{RW}$ Aurigae stars in both hemispheres was made by Glass and Penston ( $M N 167,237)$, who measured and discussed (mostly in terms of circumstellar material) JHKL magnitudes and colours. The spectral energy distributions of T Tauri stars, obtained by Kuhi $(A A S u p 15,47)$ with the aid of a photoelectric spectrum scanner in the range from 0.33 to $1.11 \mu \mathrm{m}$ show, as one of the results, an increase of reddening corrections towards earlier type which is probably caused by circumstellar material. The emission-line fluxes are also measured and discussed. At the other hand Dumont et al. $(A A 29,199)$ observed total fluxes of the $\mathrm{H} \alpha$ emission for some $20 \mathrm{~T}$ Tauri stars in the Taurus-Auriga-clouds and was led to a chromospheric model at these stars without recourse to an extended envelope, though according to the model central line reversals should be present, but are not observed in all cases. Imhoff and Mendoza (Rev. Mexicana Astron. Astrofis. 1, 25) computed luminosities for T Tauri and related objects and found that spectroscopic and photometric luminosities do not always coincide, possibly as a consequence of a peculiar extinction law acting. 
The light curves of selected Orion population stars at conventional wavelengths have been patrolled and studied at Sonneberg Observatory; special attention was paid to effects which could be of circumstellar origin. Results have been published on the 'pseudo super-giant' RR Tauri (Röbiger and Wenzel, $A N$ 295, 47). The superposition of chromospheric activities and circumstellar dust phenomena can also explain the complex character of variability of RW Aurigae (Wenzel). Mass loss in T Tauri stars is used to explain quantitatively the correlation between the amount of interstellar extinction in the surroundings of these stars and the intensity of their $\mathrm{H} \alpha$ emission (and of other emission features) (Wenzel, $A N$ 296, 183). Nandy and Pratt (ASS 19, 219 ) investigated the variability of T Tauri-like stars in NGC 2264 by means of UBVRI observations and found the range of changes less in I than in the $U B V$ region. The polarization measurements of Breger $(A p J 188,53)$ at some Orion population stars revealed considerable effects; especially unusual behaviour has been found in RY Tauri and Walker 90 of NGC 2264, both of which show time-variable polarization pointing probably to variations in the circumstellar shell.

A spectroscopic and spectrophotometric study of the Herbig-Haro object No. 1 was done by Böhm and co-workers ( $A p J 179,149)$, using four image-tube spectra of dispersions of 51 and $134 \AA \mathrm{mm}^{-1}$, taken at the 84-in. telescope of Kitt Peak National Observatory. No evidence of the presence of fast particles such as protons exciting the emissions is existing, but though Balmer decrement and line profiles agree with those of planetary nebulae the ionisation and excitation mechanisms should be of essentially another kind because of the absence of any detectable central star. K. M. Strom et al. searched for infrared sources associated with HerbigHaro objects, and Gull reports the detection of a number of new such objects $(B A A S 5,437)$.

A detailed investigation of the evolution of extremely young clusters with special emphasis put on the group properties of their Orion population members was undertaken by Gotz (summarizing paper: Veroeffentl. Sternw. Sonneberg 8, No. 3) who at present is working on properties of gas and dust shells of T Tauri stars. Herbig $(A p J 182,129)$ interpreted BD-10 4662, which is known to have flared at least four times, as a post-T Tauri star; it is a close double $\left(\mathrm{K} 5 \mathrm{p} \mathrm{V}+\mathrm{K} 7 \mathrm{p}\right.$ V) with $\mathrm{H} \alpha$ emission and $\mathrm{Li}$ I absorption. The star at $20^{\mathrm{h}} 20^{\mathrm{m}}+41^{\circ} .9$, the variability of which was discovered by Herbig, and which shows a nebular appendage, was investigated on plates of Sonneberg (Wenzel and Gessner, MVS 7, 23) and of Nantucket (Hoffleit). The object gradually brightened from $16^{\mathrm{m}} \cdot 0$ to $13^{\mathrm{m}} \cdot 5$ in the years since 1948 .

By Grasdalen et al. (PASP 85, 193) eleven dust clouds exhibiting radio $\mathrm{OH}$ lines have been surveyed for $\mathrm{H} \alpha$ emission-line stars. Eight new objects, 'most likely T Tauri stars', have been discovered. For further surveys concerning extremely young variables see the report on survey work.

At the Moscow IAU Symposium No. 67 on 'Variables in Relation to the Evolution of Stars and Stellar Systems' G. Gahm gave an introductory review paper on 'T Tauri stars and FU Orionis Stars'.

\section{CEPHEIDS \\ (J. D. Fernie)}

\section{A. Classical Cepheids}

No comprehensive review paper dealing with all aspects of current classical Cepheid research seems to have been written in the past three years, but one that does cover many areas is by Iben and Tuggle ( $A p J 197,39)$.

\section{Period-Luminosity Relation}

Papers: Iben and Tuggle (ApJ 197, 39); Kobayashi (Sci. Rep. Tohoku Univ. 57, 41); Wielen (AA Sup 15, 1); Feast (MN 169, 273); Karp (ApJ 200, 354); Fernie (IAU Symp 67, 185). The zero-point of the P-L relation appears to still be uncertain by about 0.3 mag. Wielen indicates there is now no great discrepancy between the distance scale of Cepheids determined from 
space motions and that from the cluster Cepheids. Fernie's paper deals with the advantages of the $R I$ photometric system over the $U B V$ system for discussing the P-L relation. Feast suggests that the P-L relation does not curve over at high luminosities as in the Sandage-Tammann calibration.

\section{Colours}

Papers: Schmidt ( $M N$ 170, 39P); Kelsall (BAAS 5, 16); Parsons (BAAS 5, 3); Schmidt ( $M N$ 163, 67); Feast (MN 169, 273); Karp ( $A p J$ 200, 354); Hutchinson ( $B A A S 5$ 345); Pel (AA Sup in press). It seems that there still is not universal agreement on the intrinsic $(B-V)$ colours of Cepheids, particularly at the longer periods. Several authors have raised the possibility of circumstellar reddening in Cepheids. Pel's work constitutes a very large body of data (all Cepheids brighter than $V=11.0$, south of Dec. $+15^{\circ}$, and 240 stable supergiants) on the Walraven 5-colour system. Readers are reminded there has been a considerable amount of Soviet work done in this area.

\section{Spectroscopy}

Papers: Karp ( $A p J$ 180, 895); van Paradijs ( $A A$ Sup 13, 407); Schmidt $(M N$ 167, 613); Schmidt et al. (ASS 29, 397, and $A p J$ 189, 293); Rodgers and Gingold $(M N 161,23)$; Hollars (ApJ 194, 137); McCarthy (IAU Symp 50, 30); Kobayashi and Katahira (Sci. Rep. Tohoku Univ. 56, 17); van Paradijs and de Ruiter ( $A A$ 24, 317); Gascoigne ( $M N$ 166, 25P). Several of these papers include abundance analyses of Cepheids, and indicate that the latter have solar abundances of metals to within a factor of 2 or 3. (This is of importance since Gascoigne's paper suggests that a change in $Z$ from 0.02 to 0.005 will decrease the luminosity of a Cepheid by 0.4 mag.) In particular, there are no significant differences (except possibly surface gravity) between Cepheids and non-variable supergiants of similar luminosity and colour. Among beat Cepheids, U TrA has a normal gravity for its period, but TU Cas does not and is probably not a classical Cepheid.

\section{Cepheids in Open Clusters}

Papers: Madore and van den Bergh ( $A p J$ 197, 55); Madore (AA 38, 471); Kobayashi (Sci. Rep. Tohoku Univ. 57, 41); Takeuti (PASJ 25, 567); Fernie (IAU Symp 67, 185). The first two papers report the discovery of additional Cepheids in clusters, including the important case of TW Nor in Lynga 6, which, with a period of $11^{\mathrm{d}}$, is now the longest period Cepheid known to be in an open cluster. Takeuti uses the cluster Cepheids to derive a mass-luminosity relation, as does Kobayashi. Fernie's paper deals with the $R I$ properties of cluster Cepheids.

\section{Extragalactic Cepheids}

Papers: Madore ( $A p J$ Sup 29, 219); Iben and Tuggle $(A p J 197,39)$; van Genderen $(A A$ 23, 153; and $A A$ 34, 279); Demers $(A J$ 78, 461); Chiosi and Nasi $(A A$ 35, 81); Feast $(M N 169$, 273). Probably the most important results are the continued finding of composition differences among Cepheids of different galaxies and the concomitant implications for a single universal P-L relation. Madore's work provides a uniform homogeneous body of data for both Galactic and extragalactic Cepheids, while van Genderen's papers are concerned with structure in the period-amplitude relations and the implications thereof. Demers comes to the unexpected conclusion that apparently-classical Cepheids can occur in apparently-globular clusters of the LMC.

\section{Miscellanea}

(Papers: Jacobson ( $A p J$ 191, 691); Schmidt ( $M N$ 163, 67); Thompson ( $M N$ 172, 455); Takeuti (PASJ 25, 567); Weiss and Wood ( $A A A 1,165)$; Abt and Levy $(A p J 188$, L75); Winzer 
(AJ 78, 618); Woolley and Carter $(M N$ 162, 379); Asteriadis et al. (Stars and the Milky Way System, Springer-Verlag, 1974, p. 17); Sanwal and Parthasarathy (AA Sup 13, 91); Percy (IB 983); Wielen ( $A A$ Sup 15, 1). Jacobsen has given convincing evidence of changes in the shape, systemic velocity and period of the radial velocity curve of W Sgr. On the other hand, photometric work by Asteriadis et al. and Bahner and Mavridis (private commun.) has shown no changes in the light curves of selected Cepheids over about a decade. Additional work on period changes in classical Cepheids has been or is done by Winzer, Abt and Levy, Scarfe (private commun.), Bahner and Mavridis (private commun.), and Szabados (Szeidl, private commun.). Szabados' work is extensive, covering some 80 northern Cepheids with periods less than $10^{d}$. Further work on Wesselink-method radii and the period-radius relation has been done by Woolley and Carter, Gieren (private commun.), and Evans (Univ. of Toronto PhD thesis 1974). Gieren's work concerns the important short period Cepheids SU Cas. Evans provides new data for the accurate phase-matching of velocities and photometry for 15 Cepheids. Weiss and Wood have found a weak magnetic field in W Sgr, and suggest that magnetic field strengths decrease sharply with period for variables in general. Wielen's paper provides a new kinematical analysis of Cepheids within $1 \mathrm{kpc}$ of the Sun. Percy's note and unpublished work by Fernie continue the search for low-amplitude Cepheids and/or stable stars within the instability strip. Thompson's paper applies Wesselink's surface brightness - colour relation to Cepheids, a relation currently being extended by Barnes, Evans, and Parsons (private commun.).

In general review, the most pressing broad problems in classical Cepheid observational research at present appear to be (a) The need to find an intrinsic colour scale on which most workers can agree; (b) The calibration of this scale in terms of effective temperature; (c) Further work on the shape and particularly the zero-point of the P-L-C relation, the zero-point being presently in dispute by several tenths of a magnitude; (d) The question of metallicity and its influence on the universal applicability of the P-L-C relation.

\section{B. Population II Cepheids}

As in the case of classical Cepheids, no comprehensive observational review paper has been written recently for Population II Cepheids. However, an exhaustive compilation of existing data and many references has been made by Bednarek (Univ. of Toronto PhD thesis 1975).

\section{Period-Luminosity Relation}

Papers: Breger and Bregman (ApJ 200, 343); Böhm-Vitense et al. (ApJ 194, 125); BöhmVitense $(A p J 188,571)$; Demers and Harris $(A J 79,627)$; Caputo and Castellani $(A S S 19,423)$; Bednarek (Univ. of Toronto PhD thesis 1975). The P-L relations derived in these papers differ considerably in slope and zero-point, and the absolute magnitudes they predict, while agreeing reasonably well at short periods, can differ by a magnitude at longer periods. Kwee's earlier suggestion $(B A N 19,374)$ that there are different $\mathrm{P}-\mathrm{L}$ relations for Cepheids with crested light curves compared to those with flat-topped light curves does not seem to have been followed up, except by Bednarek.

\section{Other Work}

Papers: Coutts (IAU Coll. 21, 145); Gehrz and Hackwell ( $A p J$ 193, 385); Stobie (Obs. 93, 111); Petersen ( $A A$ 27, 89); van Agt (IAU Coll. 21, 35); Landolt (PASP 87, 373); Hutchinson (PASP 85, 119). Coutts' work deals with period changes in Population II Cepheids. Gehrz and Hackwell provide new infrared measurements for 10 stars, finding no infrared excesses. Stobie's paper investigates the existence of a Hertzsprung progression in Population II Cepheids, and the application of Christy's 'bump' method to determining their radii. Petersen analyzes doublemode Cepheids to find their masses, obtaining values in the vicinity of 1 solar mass. Van Agt's paper reviews variables in dwarf spheroidal systems. Landolt and Hutchinson report work on V553 Cen and IC 1613 no. 39 respectively. Kwee (private commun.) reports extensive UBV photometry of 15 field variables with periods between 1 and 3 days, finding substantial period variations in some cases. 
In general review, it seems clear that significant advances in Population II Cepheid research will require improved observational data. In particular, many current papers rely on dubious transformations of old photographic work done on globular cluster variables, and it is most desirable that the very large southern hemisphere reflectors now becoming operational be applied to acquiring more accurate homogeneous data for these stars.

\section{DELTA SCUTI VARIABLES AND DWARF CEPHEIDS}

\section{(J. R. Percy and J. D. Fernie)}

\section{A. Delta Scuti Variables}

A comprehensive review was published by Baglin et al. $(A A 23,221)$. Other general studies and surveys include the following: (i) Surveys for new $\delta$ Scuti stars by Breger $(A A 22,247)$ and by Percy $(J R A S C 67,139)$; (ii) Observations of several previously known or suspected $\delta$ Scut stars by Elliot $(A J$ 79, 1082); Fesen $(P A S P$ 85, 732); Jorgensen and Norgaard-Nielsen $(A A S u p$ 19, 235); Valtier et al. ( $A$ A Sup 18, 235); Warman et al. Rev. Mexicana Astron. Astrofiz. 1, 143); Gupta (Obs 93, 192); Gupta and Bhatnagar (IB 751 and 778); (iii) A determination of the $Q$-values and pulsation modes of known $\delta$ Scuti stars by Breger and Bregman $(A p J 200,343)$, in which a clear separation of fundamental and overtone pulsators was found in the H-R diagram.

Several investigators have studied the complex light curves of $\delta$ Scut stars. Gray and Desikachary $(A p J 181,523)$ developed a new approach to periodogram analysis, and applied it to DQ Cep and to 44 Tau $(A A 27,331)$. Smyth et al. $(M N 171,143)$ analysed observations of three $\delta$ Scuti stars, finding that in HR 242 the component periods are a function of the epoch of observation and change on a time scale as short as a few weeks. Other analyses are contained in the papers cited above by Gupta and Gupta and Bhatnagar, and additional work on other stars continues at Uttar Pradesh (Sinvhal, private commun.). Percy $(A J 80,698)$ has pointed out the advantages of the maximum entropy method of spectral analysis for the study of these stars. In many $\delta$ Scut stars the component periods cannot be interpreted in terms of fundamental and overtone modes of radial pulsation.

The relationship between rotation, abundance anomalies, and $\delta$ Scuti pulsation has been a subject of great interest. Breger showed in 1970 that no Am stars are $\delta$ Scuti stars, with the exception of $32 \mathrm{Vir}$. This star, however, is also a spectroscopic binary, and Breger (BAAS 7 , 404) suggests that one component may be an Am star, the other a $\delta$ Scuti star. Percy $(A J \mathbf{8 0}$, 698) has shown that $\delta$ Scuti pulsation is infrequent in Ap stars, but that the Ap star 21 Com definitely is a $\delta$ Scuti variable. Walker (private commun.) has shown that the $\lambda$ Boo star 29 Cyg is a $\delta$ Scuti star. Morgan and Abt $(A J 77,35)$ have noted some of the subtle spectral peculiarities in known $\delta$ Scuti stars; Breger and Kurtz $(B A A S 5,440)$ have commented on this same problem. Joshi and Rautela $(A A 29,155)$ have undertaken detailed studies of the energy distribution, effective temperatures, gravities, masses, and radii of beta Cas and upsilon UMa, and similar studies for 14 other $\delta$ Scuti stars are in progress. A model atmosphere analysis of $20 \mathrm{CVn}$ has been completed by Ishikawa ( $P A S J 271)$, who finds that while $\mathrm{Sr}, \mathrm{Zr}, \mathrm{Ba}, \mathrm{Ce}$, and Eu are over-abundant by factors of 7 to 16 compared to the Sun, the overabundance is not as extreme as in Am stars. Leung (private commun.) reports the existence of a considerable collection of $127 \AA \mathrm{mm}^{-1}$ spectrograms of $\delta$ Scuti stars, soon to be classified on the MK system. Leung $(A J 79,626)$ has also obtained radial velocities of $\delta$ Del.

\section{B. Dwarf Cepheids}

A summary of the findings of an extensive study of dwarf Cepheids is given by McNamara and Langford (PASP 87, 505). Warner and Nather ( $M N 156,315$ and 321) have applied the technique of high-speed photometry to these stars, and Van Citters has begun to use a Griffintype radial velocity spectrometer to study their velocity curves at high time resolution. The problem of interpreting secondary periods in these stars is well illustrated by a series of papers 
in $A A 17,148, A A 27,161$, and $A A$ Sup 21, 177. Percy $(A A$, in press) has shown that the period of CY Aqr changed abruptly in 1951, and Barnes and Moffett $(A J \mathbf{8 0}, 48)$ have shown that the phase-shift diagram for SZ lyn is consistent with its being a binary star with a 3.14-year period. Epstein $(A J 78,83)$ has made extensive observations of dwarf Cepheids and RR Lyrae stars in the uvby system (see also McNamara and Langford above).

\section{Integrated Studies of Dwarf Cepheids and Delta Scuti Stars}

There is no firm evidence that $\delta$ Scuti stars and dwarf Cepheids are separate species; the latter may simply be large-amplitude versions of the former. The star $1 \mathrm{Mon}$, for instance, $(P A S P 85,410)$ varies in amplitude from $0 \mathrm{~m} 03$ - typical of $\delta$ Scuti stars - to 0 35 , which is typical of a dwarf Cepheid. Breger ( $A p J$, in press) has shown that the dwarf Cepheid AD CMi has a Population I mass, and Bessell (IAU Symp. 59,63) has found that the $\log g$ values for dwarf Cepheids and $\delta$ Scuti stars are similar. Chevalier (IAU Colloq. 17, 1) has discussed the same problem, and similar evidence is provided by McNamara and Langford (cited above).

\section{NOVAE AND NOVA-TYPE BINARIES}

\section{(J. Smak)}

Novae, U Gem-type, and nova-like variables have been discussed during several symposia and colloquia devoted to broader subjects, such as stellar evolution (IAUSymp. 59 and 66) or close binary systems ( $I A U S y m p$. 73). Complete references to all such meetings, all other individual papers, and - in particular - to the original observational data, can be found in Astronomy and Astrophysics Abstracts.

Observations of novae and $U$ Gem-type variables at their minima continued to provide information about their basic physical properties. Among them, masses were discussed by Warner $(M N 162,189)$ and Robinson $(A p J$, in press). New pe observations of $Z$ Cha (Warner, $M N 168,235$ ), covering also several outbursts of this dwarf nova, showed that - like the case of $\mathrm{U}$ Gem itself - the outburst consists of a major brightening of the central parts of the disk. Extensive pe observations of another southern dwarf nova VW Hyi (Vogt, $A A$ 36, 369; Warner, $M N 170,219)$ led to a discovery of periodic humps in the light curve during the characteristic super-maxima of this object. The period of these humps is somewhat longer than the orbital period and the physical nature of the phenomenon is not clear. Radiation of many old novae and $U$ Gem-type objects was measured in a wide range from UV (Holm and Gallagher, $A p J$ 192, 425; Gallagher and Holm, $A p J$ 189, L123; Wu, IAU Symp. 73) to IR (Feast, Glass, $M N$ 167, 81; Szkody, $A p J$ 192, L75). Very soft X-rays from SS Cyg were also detected (Rappaport et al., $A p J$ 187, L5; Heise et al., in press). These new data show that the spectral energy distributions of novae and $U$ Gem-type objects is not drastically different from those of normal stars (with $T$ $=1-3 \times 10^{4} \mathrm{~K}$ ) and, while there are some peculiarities, there is no convincing evidence for temperatures in excess of $3 \times 10 \mathrm{~K}$, even in the case of dwarf novae observed at their outbursts.

The short-period, coherent oscillations observed during the outbursts of dwarf novae were studied more extensively but remain rather puzzling (for a recent review, $\mathrm{Cf}$. Warner, $I A U$ Colloq. 29). These oscillations resemble somewhat the $71-\mathrm{s}$ oscillations in DQ Her and the transient 30-s oscillations in UX UMa (Nather and Robinson, $A p J 190,637$ ). The evidence now available for DQ Her and UX UMa excludes the possibility that these oscillations could be due to pulsations of the white dwarf. In a number of papers therefore new suggestions appeared connecting the periodicity with the rotation of the white dwarf (e.g. Bath et al., MN 166, 113; Herbst $e t a l ., A p J 193,679)$ and the light variations with the scattering or absorption remission of the radiation of the white dwarf in the circumstellar disk (cf. Herbst et al., op. cit.; Katz, $A p J$ 200, 298). Discovery of variable linear and circular polarization (Kemp et al., ApJ 193, L11; Swedlund et al., ApJ 193, L15) is of great interest in this respect, but so far the results are inconsistent with any of the possible models, most likely due to low accuracy of the measurements. It is likely that once the oscillation mechanism in DQ Her and UX UMa is well understood, the same mechanism or its modification will be applicable to the oscillations in dwarf novae. 
Observational data have been collected and analysed for a number of novae at outburst. The slow Nova HR Del 1967 was subject of particularly numerous investigations: A long series of pe observations was made by Tempesti (in prep.) while spectroscopic studies of the expanding envelope included papers by Andrillat et al. $(A S S$ 31, 169), Babayev $(A Z 52,48)$, Friedjung et al. $(A S S$ 25, 433), Sanyal ( $A p J$ Sup 28, 115), and an extensive series by Malakpur $(A A 24,125$; 28, 393; ASS 24, 577; 27, 467). Nova Cyg 1975, one of the brightest novae ever observed, is likely to get a particularly detailed and complete coverage of its outburst. Important, far UV photometry of Nova FH Ser 1970 (Gallagher and Code, $A p J$ 189, 303) showed that the bolometric flux of this object remained essentially constant for nearly 2 months beyond visual maximum.

The theory of the nova outburst, based on the thermonuclear runaway mechanism, has reached the stage where a more detailed comparison with observations is possible. Recent models by Starrfield et al. (ApJ Sup 261;ApJ 192,647) led to a conclusion that a significant overabundance of CNO is needed to produce a nova type explosion; such ioverabund ances have already been noticed in the expanding envelopes. On the other hand, it appears that this mechanism is incapable of explaining a dwarf nova phenomenon. Here the most promising mechanism is connected with the possibility of a sudden instability in the disk, followed by an increased accretion of the material onto the white dwarf (Osaki, PAS J 26, 429; Bath et al., MN $169,447)$.

\section{SUPERNOVAE}

\section{(J. Smak)}

Supernovae and supernova remnants have been discussed during several symposia and colloquia and in a number of review articles. Meetings devoted exclusively to supernovae included: Conference on Explosive Nucleosynthesis, Austin, Texas (ed. by Schramm and Arnett, Univ. of Texas Press, Austin, 1973), Supernovae and Supernova Remnants, Lecce, Italy (ed. by Cosmovici et al., Reidel, 1974), while the most important reviews were: Arnett (ARAA 11, 73), and Oke and Searle $(A R A A 12,315)$. An atlas of spectra of supernovae by Greenstein and Minkowski $(A p J 182,225)$ also falls into this category. Complete references to all such reviews, to individual papers, and - in particular - to the original observational data, can be found in Astronomy and Astrophysics Abstracts.

A considerable progress has been made in the studies of dynamics, and chemical composition of the expanding supernova envelopes (cf., e.g. Friedjung, $A A$, in press; Gordon, $A A$ 29, 123; $A p J$ 198, 765; Kirschner and Kwan, $A p J$ 197, 415; Mustel, $A Z$ 50, 1121 ; Shklovsky, $A Z$ $51,3)$ and in the theory of the supernova origin and explosion mechanisms (cf. Texas Conference). Supernovae in binary systems have received a considerable attention (e.g. Cheng, $A S S 31$, 49; Habazin, $A Z$ 52, 57; Sutantyo, $A A$ 41, 47). In an application to extragalactic research, Kirshner and Kwan $(A p J 193,27)$ successfully applied the Baade's method to supernovae in NGC 1058 and $M 101$, and obtained an independent estimate of the Hubble constant, $H_{0}=60$ $\pm 15 \mathrm{~km} \mathrm{~s}^{-1} \mathrm{Mpc}^{-1}$.

\section{VARIABLE STARS IN THE MAGELLANIC CLOUDS}

\section{(S. C. B. Gascoigne)}

C. J. Butler (AASup 1975) and Les Connolly (to appear) have observed a substantial number of cepheids in each cloud, on Schmidt plates with calibrating stars measured photoelectrically. B. Madore (ApJ Sup 29) has made UBV photoelectric observations of $22 \mathrm{LMC}$ and 15 SMC cepheids. All writers have discussed the P-L-C and period-amplitude relations, Madore concluding that the largest amplitudes occur on the red side of the instability strip. E. M. Lindsay ( $M N$ 166,$703 ; M N 169,343$ ) has found periods for 33 new variables in the LMC. 
Feast $(M N 169,273)$ has found that the longest period cepheids are too red, by 0.2 to 0.4 in $B-V$, for their spectral types; this is possibly because of (circumstellar) absorption. J. W. Robertson $(A p J 185,817)$ has completed evolutionary tracks for 3.5 and $4.0 M_{\odot}$ stars which suggest that the excess of short-period cepheids in the SMC may be a consequence of mild metal deficiency. S. Gascoigne $(M N 166,25 \mathrm{p})$ has calculated the effect of such a deficiency on the luminosities and colours of cepheids.

J. A. Graham gives periods and other data for 18 RR Lyraes near NGC 1835 in the LMC ( $A J$ 19, 363; with Ruiz), and for 77 near NGC 121 in the SMC (PASP, Oct 1975, to appear). In the latter field the period distribution resembles that in Leo II. The SMC variables peak quite sharply at $B=20^{m}$, and appear to be distributed rather evenly over the SMC.

A. Ardeberg and M. de Groot $(A A 26,563)$ discuss the $U B V$ observations for nova Dor 1971a. Feast and B. L. Webster $(M N 168,31$ p.) describe five bright LMC variables, two of which are probably symbiotic stars and two VV Ceph stars.

\section{PROGRESS IN FLARE STAR RESEARCH}

\section{(J. E. Grindley and Sir Bernard Lovell)}

During the past three years investigation of flare stars, both observational and theoretical, has remained an active field of research. We shall briefly summarize some of the recent observational studies of UV Ceti stars in the radio, optical and X-ray bands as well as some recent theoretical interpretations.

Observations of the radio emissions from flare stars have been continued at Jodrell Bank in collaboration with simultaneous optical observations at a number of observatories. The most important result was the simultaneous radio and optical observations of a large flare on UV Ceti (Lovell, Mavridis and Contadakis, 1974). The flare, recorded photoelectrically at the Stephanion Astronomical Station in Peloponnese, was greater than 4.55 mag. in $B$ colour. The radio flare on $408 \mathrm{MHz}$ reached a peak $11 \mathrm{~min}$. after the peak phase of the optical flare. The sharp front of the optical flare preceded that of the radio flare by $8 \mathrm{~min}$. The total energy output was estimated to be $10^{31}$ to $10^{32} \mathrm{erg}$ (equivalent to that of the quiescent photospheric continuum). The ratio of optical to radio energy output was found to be $10^{5}$, similar to that for large solar flares.

On the basis of these results, Kahn (1974) developed a theoretical model for the flare assuming that the energy of the flare is released during the reconnection of the lines of force in a magnetic field, as in the solar case. A bubble of plasma with magnetic field then expands into the corona of the star, preceded be a shock wave. After about $8 \mathrm{~min}$ the bubble reached the level in the corona at which $408 \mathrm{MHz}$ radiation could be propagated. The initial linear dimension of the flare was calculated to be about $2.5 \times 10^{9} \mathrm{~cm}$, and the magnetic field $600 \mathrm{G}$. The $408 \mathrm{MHz}$ radiation was propagated at a height of $1.4 \times 10^{10} \mathrm{~cm}$ in the corona when the linear dimensions had expanded by 5.6 times and the magnetic field had dropped to $20 \mathrm{G}$. The model gives a satisfactory account of the main features of the optical-radio emissions found in this and other flares and enables the amount of energy carried away by the stellar wind to be estimated.

This new evidence for the basic similarity of the flares on the Sun and the M type stars led Lovell (1974) to investigate the extent to which flare stars might contribute to the flux of low energy cosmic rays in the Galaxy. Adopting the factor, determined for the Sun, for the conversion of flare energy to cosmic ray energy, estimates have been made of the fraction of galactic cosmic rays which may be generated in the flares on the $M$ and $K$ type stars. It is shown that the stars may be a major source of the galactic cosmic rays for energies from $10^{6}$ to $3 \times 10^{8} \mathrm{eV}$ and that the $\mathrm{K}$ type stars may contribute one-fifth of the total cosmic ray energy up to $10^{9} \mathrm{eV}$.

A high sensitivity radio survey from Arecibo of several flare stars was reported by Spangler et al. (1974a). Flares of only a few tenths a flux unit and duration a few tens of seconds were observed at $318 \mathrm{MHz}$, such that the detection rate of small radio flares is apparently comparable to that of optical flares in the B-band with an intermediate size telescope. Another significant result of Arecibo observations at $430 \mathrm{MHz}$ of Spangler et al. (1974b) was the first detec- 
tion of polarization in a flare of AD Leo. This result suggests the path integral of the product of magnetic field and particle density in this flare was comparable to solar values.

Results of an extensive program of photographic observations of the flare star population in the Pleiades have been presented by Ambartsumian et al. (1973). A total of 415 flare stars were reported and statistical arguments given that the actual total may exceed 1000. Bopp and Moffett (1973) have presented results of simultaneous high-speed photometry and time-resolved spectra of two large flares of UV Ceti. The observations suggest that flares are complex and involve multiple mechanisms for energy dissipation, including possible shock wave energy transport. Results of three-color photometry of EV-Lac were reported by Flesch and Oliver (1974) in which dips in the $r$-band intensity were observed during flares. Weaver (1974) obtained photometric measurements of many of the rapid variables in the Coalsack and found they were not UV Ceti spectral type. Finally, Boesgaard and Hagen (1974) have derived an age $\left(>10^{9} \mathrm{yr}\right)$ for $a$ Cen, associated with the flare star $a$ Cen $C$. This appears inconsistent with earlier estimates of flare star age limits $\left(<4 \times 10^{8}\right)$.

Considerable attention has been paid in the past three years to the possibility of detecting $\mathrm{X}$-rays from UV Ceti flares. Grindlay et al. (1973) conducted a search for X-rays from AD Leo using Uhuru data and limited the $2-6 \mathrm{keV}$ luminosity to be $<10^{30} \mathrm{erg} \mathrm{s}^{-1}$ from an observation $\sim 3$ min after a $\Delta \mathrm{m}_{\mathrm{u}} \sim 4$ optical flare. Hudson and Tsikoudi (1973) obtained simultaneous X-ray $(>8 \mathrm{keV})$ coverage with OSO 3 of a number of optical flares and limited the luminosity to be $<3 \times 10^{33} \mathrm{erg} \mathrm{s}^{-1}$ for a $\Delta \mathrm{m}_{\mathrm{u}} \sim 1.5$ flare of $\mathrm{YZ} \mathrm{Cmi.} \mathrm{Crannell} \mathrm{et} \mathrm{al.} \mathrm{(1975)}$ have also set an upper limit $\left(<10^{31} \mathrm{erg} \mathrm{s}^{-1}\right)$ for the X-ray luminosity of a $\Delta \mathrm{m}_{\mathrm{u}} \sim 0.9$ flare of YZ Cmi observed by OSO 7. However, in the past year X-ray flares have been detected from both YZ Cmi and UV Ceti with luminosities below these earlier upper limits. This has resulted from observing programs by the Astronomical Netherlands Satellite (ANS) on YZ Cmi in October 1974 and UV Ceti in January 1975. During both periods an extensive campaign of coordinated radio and optical coverage was carried out, with the most complete coverage obtained for UV Ceti. Radio observations for both programs were conducted at Jodrell Bank by Prof. B. Lovell and for UV Ceti by Dr P. Feldman at Penticton Radio Observatory. Likëwise, optical coverage was provided by Prof. P. Chugainov at the Crimean Observatory; Drs S. Cristaldi and M. Rodono, Catania Observatory; Dr E. Groth, Princeton Observatory; Mr K. Ichimura, Okayama Observatory; Dr W. Kunkel, Cerro Tololo Observatory; Dr L. Mavridis, University of Thessaloniki; Messrs. B. Marion and W. Walker, Auckland Observatory; Dr T. Moffett, McDonald Observatory; Dr R. Stagni, Asiago Observatory; Dr N. Vidal, Siding Springs Observatory; and observers of the American Association of Variable Star Observers.

A large X-ray flare was detected (Heise et al., 1974) from $\mathrm{YZ} \mathrm{Cmi} \mathrm{both} \mathrm{at} \sim 0.25 \mathrm{keV}$ $\left(L \sim 3 \times 10^{29} \mathrm{erg} \mathrm{s}^{-1}\right)$ and $\sim 1-7 \mathrm{keV}\left(L \sim 4 \times 10^{30} \mathrm{erg} \mathrm{s}^{-1}\right)$. Unfortunately, there was no known optical (or radio) observation of this flare. During the ANS observations of UV Ceti, a very large optical flare $\left(\Delta \mathrm{m}_{\mathfrak{u}} \geq 6\right)$ was observed by Kunkel and also $\left(\Delta \mathrm{m}_{\mathrm{v}} \simeq 3\right)$ by Groth. The ANS detectors registered a soft $(\sim 0.25 \mathrm{keV})$ X-ray flare of peak luminosity $\sim 6 \times 10^{28}$ $\mathrm{erg} \mathrm{s}^{-1}$; an upper limit of $2 \times 10^{30}$ erg s $\mathrm{s}^{-1}$ was obtained in the $1-7 \mathrm{keV}$ range (Heise $e t$ al., 1975). Two small optical flares $\left(\Delta \mathrm{m}_{\mathrm{h}} \sim 0.5\right)$ were also observed by Ichimura and one additional flare $\left(\Delta \mathrm{m}_{\mathrm{u}} \sim 1.2\right)$ by Kunkel during the times of ANS coverage of UV Ceti, and upper limits at $\sim 0.25 \mathrm{keV}\left(\sim 2-9 \times 10^{-11} \mathrm{erg} \mathrm{cm}^{2} \mathrm{~s}^{-1}\right.$ and at $\sim 2-6 \mathrm{keV}\left(\sim 0.6-2 \times 10^{-9} \mathrm{erg} \mathrm{cm}^{-2} \mathrm{~s}^{-1}\right.$ were obtained (Heise et al., 1975).

These results suggest that X-ray flares may accompany optical flares with luminosity ratio $L_{\mathrm{x}} / L_{\text {opt }} \sim 10^{-2}$. This is most consistent with a prediction of Kahler and Schulman (1972) based simply on scaling from the $L_{\mathrm{x}} / L_{\text {opt }}$ ratios observed in solar flares. A similar scaling from observed solar radio/X-ray flare luminosities (Crannell et al., 1974) leads to higher UV Ceti X-ray flare luminosities. Mullan (1975) has proposed yet another optical scaling model that, on the basis of the two ANS events, provides better agreement. Clearly more observations of X-ray flares are needed, though it is possible UV Ceti flares are an important contribution to the soft X-ray background. 


\section{REFERENCES}

Ambartsumian, V. A., Mirzoyan, L. V., Parsamyan, E. S., Chavushyan, O. S., Erastova, L. K., Kazaryan, E. S., Oganyan, G. B., and Yankovich, I. I.: 1973, Astrofiz. 9, 461.

Boesgaard, A. M. and Hagen, W.: 1974, ApJ 189, 85 .

Bopp, B. W. and Moffett, T. J.: 1973, $A p J 185,239$.

Crannell, L. J., McClintock, J. E. and Moffett, T. J.: 1974, Nat 252, 659.

Flesch, T. R. and Oliver, J. P,: 1974, ApJ 189, L127.

Grindlay, J. E., Koch, D., and Weekes, T. C.: $1973, B A A S 5$, 381.

Hudson, H. S. and Tsikoudi, V.: 1973, Nat PS 245, 88.

Kahler, S. and Schulman, S.: 1972, Nat PS 237, 101.

Kahn, F.: 1974, Nat 250, 125.

Heise, J., Brinkman, A. C., Schrijver, J., Mewe, R., Gronenschild, E., den Boggende, A., Grindlay, J., Schnopper, H., Schreier, E., Gursky, H., and Parsignault, D.: 1974, IAU Circ No. 2731.

Heise, J., Brinkman, A.C., Schrijver, J., Mewe, R., Gronenschild, E., den Boggende, A., and Grindlay, J.: 1975, ApJ (Letters), (in press).

Lovell, B.: 1974, Phil. Trans. Roy. Soc. London, A277, 489.

Lovell. B., Mavridis, L. N., and Contadakis, M. E.: 1974, Nat 250, 124.

Mullan, D. J.: 1975, submitted to $A p J$.

Spangler, S. R., Shawhan, S. D., and Rankin, J. M.: 1974a, ApJ 190, L1 29.

Spangler, S. R., Rankin, J. M., and Shawhan, S. D.: 1974b, ApJ 194, L43.

Weaver, W. B.: $1974, A p J 189,81$.

\section{REPORT ON VARIABLE STARS IN GLOBULAR CLUSTERS}

\section{(H. B. Sawyer Hogg)}

The papers presented at IAU Colloquium No. 21, 1972 have been published in 'Variable Stars in Globular Clusters and Related Systems', (ed. by J. D. Fernie), D. Reidel, 1973 and form a valuable and comprehensive summary of various aspects of this work.

Since then some new developments merit special comment. First is a totally new type of variable object in globular clusters, - variable X-ray sources, revealed by observations from the Uhuru and OSO-7 satellites. Four such sources are now known and all are variable. Second, the red variables are gaining increasing importance in globular clusters. More and more are being found, and they are numerous enough to change noticeably the earlier percentages given for the proportion of RR Lyrae stars in these clusters. Third, earlier ideas that red variables in clusters would have periods less than 220 days have been revised, as stars with longer periods, apparently cluster members, have been found.

\section{A. Catalogues}

The Third Catalogue of Variable Stars in Globular Clusters, comprising 2119 entries, by H. Sawyer Hogg (Publ. David Dunlap Obs. 3, No. 6) appeared a few months after the last IAU General Assembly. Information which was published in time for inclusion in the catalogue is not included in this report. This writer now requests information pertinent to the Fourth Catalogue, slated to go to press in 1978 , and acknowledges the painstaking help of $\mathrm{Dr} \mathrm{H}$. Wilkins of La Plata.

\section{B. Discovery of New Variables, and Periods Determined for Some}

In most cases new variables are now being found only a few at a time in clusters under investigation. In NGC 18514 new variables have been found by Liller ( $A p J$ in press), who has determined periods for $11 \mathrm{RR}$ Lyrae stars and notes that one variable is very red. None of these variables seems to have any connection with the X-ray source. She also has a new RR Lyrae variable in 2298 and 3 new variables in 2808, and is working on another X-ray cluster, 6624. 
She states that 4372 has been adequately hunted. Terzan and Rutily ( $A A$, in press) have 57 variables in or around 5 clusters: NGC 4590 (4), 6401 (22), 6628 (26), 7099 (1), Terzan 1 (4), and comment that no more are to be found in 1904 and 6304. In 5139 Cannon and Stobie $(M N 162,207,267)$ have 9 probable variables with amplitudes greater than 0.06 mag., plus 2 field red variables, and in 6752 one new small amplitude red variable.

Around 5272 Meinunger (MVS 6, No. 3) has 8 new variables, 4 RR Lyrae and 4 slow, not red, noting one slowly variable object (IB 738). In NGC 6273 Coutts, Sawyer Hogg and Thompson have one new variable ( $B A A S$, in press) bringing the total in the cluster to 5 , of which 2 are RR Lyrae type. Light curves have been determined for 3 Population II Cepheids. In NGC 3201 Wilkens finds that HW 10 is actually a new variable, and not the same as Var. 39, as published. In 6402 Wehlau, Davis and Potts $(I B$ 859) have 5 more new variables, which, with the 12 they announced earlier, bring the total in the cluster to 93. In 6638 Sawyer Hogg, Rutily and Terzan have 26 new variables, 17 in cluster, 9 in field ( $C R$ Ser. B, 279, 333), and Bally (Thesis, Université Lyon) has light curves for $5 R_{R_{c}}$ variables in the cluster, and 8 in the environs.

Menzies $(M N 168,177)$ has completed his long study of variables in NGC 6723 , bringing to 12 the number of new variables found, 10 RR Lyrae stars and 2 red variables. Periods have been found for 29 RR Lyraes. The mean periods are 0.291 days and 0.540 days on a $5 / 24$ ratio of $c$-type to $a b$-type, an Oosterhoff Type I cluster.

In the Sagittarius object Terzan 5, Spinrad, Smith and Harlan $(A p J 192,405)$ have found 2 very red variables and one blue, probably a foreground object, on infrared photographs taken at Lick and Cerro Tololo. Mira periods of 227 days and 245 days are determined, and both stars are considered members. However, they do not prove whether Terzan 5 is a galactic globular cluster or a dwarf galaxy because the distance derived is greatly affected by absoprtion. According to Wing, Warner and Smith $(A p J 179,135)$ Terzan 5 , IRC -20385 is probably a normal globular cluster at $8 \mathrm{kpc}$ distance, súffering $9.4 \mathrm{mag}$. visual absorption.

\section{Periods or Types Determined for Known Variables}

The determination of long Mira periods in globular clusters by Andrews, Feast, Lloyd Evans, Thackeray and Menzies (Obs 94, 133) changes the notion that these stars do not exist in globular clusters. Earlier periods of Lloyd Evans and Menzies are confirmed. The period of Var. 3 in NGC 5927 is 310 days and radial velocity measures suggest cluster membership. Var. 4 in 6553 has a period of 265 days, membership probable. The period for Var. 5 in NGC 6637 is 195 days.

There is a marked improvement in our understanding of the variables in the Hercules cluster M 13. Russev ( $P e r Z v 19,181$ ) has published periods for 3 Cepheids, 2 RR Lyrae stars and 2 slow variables, including a new period for Var. 15, 140.3 days. Fuenmayor and Osborn (IB 952) confirm a variable suspected by Russev, with a range of 0.4 mag., and an irregular period near 39 days. Osborn $(M N 162,91)$ has cleared up an earlier confusion regarding Vars. 5 and 9 , both $R_{R_{c}}$ stars. With his collaborators (Ibanez and Osborn, IB 769) he has derived a different period for Var. 7 , also an $\mathbf{R}_{\mathbf{c}}$ type and reports $(I B 798,849)$ that Var. 3 and Var. 16 show no variation.

In NGC 1261 periods have been determined for the first time. Wehlau, Davis and Demers (IB 871) report 6 RR Lyrae periods from 0.53 to 0.61 days. Cacciari (IB 905) has found new periods for 3 RR Lyraes in 5466, 6333, and 6426, and has improved the magnitudes for the period-amplitude relation for $13 \mathrm{RR}_{\mathrm{c}}$ type variables in M 3 ( $A A$ Sup 16,63). Goranskij (Astr. Cirk. Izdav. Bjuro. Astr. Soobsc. 767; 832) has determined periods in NGC 5024 for 7 RR Lyrae stars whose periods were previously unknown, one at 0.35 day, the others greater than 0.62 day. In 5634 Liller and Sawyer Hogg have RR Lyrae periods for 6 of the known variables almost ready for publication. A fuller investigation of variables in and around NGC 6522 (Hartwick, Hesser and Hill, $A p J$ 174, 573; Plaut, $A A$ 26, 317) has corrected many earlier periods which were fictitious, and has produced a different frequency distribution. In NGC 6752 a period of a Population II Cepheid for Var. 1 has been determined independently by Wesselink (IB 895) and Lee (Obs 94, 74), in agreement to the 3rd decimal, 1.378, a star described by Cannon and Stobie as almost certainly a cluster member. 
In the variable-rich cluster IC 4499 period determination is under way, with no periods yet published for individual stars. Fourcade, Laborde and Arias, discoverers of the variable richness of this cluster show ( $A A$ Sup 18, no. 3) that most of the variables are RR Lyrae stars from their position on the color-magnitude diagram. Coutts, Dickens, Epps and Read (ApJ 197, L45) have periods of 35 variables. The mean period of $28 a b$-type variables is 0.582 , similar to Oosterhoff Group I clusters, but with a substantially longer period. They suggest that the distinction between Oosterhoff groups may not be as marked as earlier supposed. Coutts is working with Rosino and Pinto on periods of variables in NGC 6864. She is also accumulating material each year at Las Campanas to follow closely period changes in $\omega$ Centauri, M 5 and NGC 6171 . In NGC 6934 Harris and Racine $(A J 78,242)$ show that their $c-m$ diagram with an even population of stars all along the horizontal branch, like M 3, explains the large number of variables. Var. 15, right at the tip of the giant branch, is probably a semiregular or long period variable. All the others are RR Lyrae type. The Sandages (A. and M.) have light curves for 60 variables in M 15 from Hale Observatory photographs.

Computer programs have been designed in many places helpful for the determination of periods, such as that described by Kurochkin $(\operatorname{Per} Z v 19,117)$.

\section{Period Changes}

Szeidl (Budapest Mitt., No. 63) in his second paper on M 3 publishes observations for 17 variables and light curves for most of them. His earlier results are confirmed, that most variables show period changes and that lengthening periods about equal in number the shortening periods. In M 14 Wehlau, Conville and Sawyer Hogg ( $A J$, in press) for 32 variables well observed over 42 years find a distribution of changes similar to those found by Szeidl in $M 3$ and Coutts in M 5, other Oosterhoff Type I clusters. However, Wesselink $(A A$ 36, 163) in a study of 12 variables in M 15 finds that 5 periods are constant, 6 are increasing and only one is decreasing.

Period changes have long been considered in evolutionary theories. Laskarides $(A S S 27,485)$ computes that evolution at a continuous mass loss rate of $1 \times 10^{-10}$ solar masses per year gives both positive and negative period variations of the order observed, 0.120 days per million years. Observed period variations in general are lower in absolute magnitude for clusters with moderate metal deficiency, and much higher for the extreme metal deficient.

\section{E. Field Variables and Significant Stars}

The determination of cluster membership is of increasing importance. Certain stars carry special significance. In $\omega$ Cen Geyer (Variable Stars and Stellar Evolution ed. by Sherwood and Plaut, p. 559, 1975) shows the UV excess for the eclipsing binary Var. 78 is similar to that of the cluster RR Lyrae stars, thereby confirming membership earlier indicated by proper motions of Dickens and Sanders. However, Naylor and Newell $(B A A S 5,386)$ find that the upper mass limits from evolutionary considerations are much smaller than the minimum mass values computed from the work of Geyer or Sistero, and cast doubt on membership. The eclipsing star Var. 65 in NGC 3201 is definitely a field star, according to Geyer. Hartwick $(P A S P 87,77)$ states that if the RR Lyrae variable No. 3, $P=0.4884$ days is confirmed as a member, then 6553 is the most metal rich cluster found to date with an RR Lyrae star. Zinn $(A p J 193,593)$ has a remarkable UV-bright star in NGC 5466. Var. 19, with period 0.82 days is brighter than the horizontal branch in the cluster by $1.8 \mathrm{mag}$. Its radial velocity is consistent with membership, but proper motions are not yet available. In M 80 Harris and Racine $(A J 79,472)$ have recomputed the absolute magnitude of the Nova T Sco 1860 from the modulus obtained from their $c-m$ diagram. At maximum its $M_{\mathrm{v}}=-8.5 \pm 0.4$, quite in keeping with cluster membership. The decay time places it in the relation for galactic novae. Mallia $(A A 41,103)$ confirms that the Mira star, Var. 1 in NGC 6397 is a non-member.

Wilkens of Cordoba while working on a project of the diameters of globular clusters from variables, writes that a number of stars listed as field variables in the Third Catalogue should really be included as members. 


\section{F. Various Correlations}

\section{RR Lyrae Stars}

Caputo and Castellani in a series of papers ( $A S S$, in press) have computed that the spread in luminosity among RR Lyrae stars results from a function of their original chemical composition. $\omega$ Cen is more helium rich than $M 3$, and no mass loss is needed to account for the characteristics. Castellani ( $A A$, in press) finds that the observed period distribution of the RR Lyraes in clusters is correlated with the transition line between $c$ and $a b$-type pulsators. Observations strongly support that differences in evolutionary directions cause the Oosterhoff dichotomy. Earlier Thänert $(A N 292,251)$ found that the mean value of masses, luminosities and radii of RR Lyrae variables decrease with increasing age of clusters, while the periods become larger and the centers of the RR Lyrae gap bluer.

A new calibration of Preston's metal abundance indicator $\Delta \mathrm{S}$ with $\mathrm{Fe} / \mathrm{H}$ ratio has been established for RR Lyraes, 47 in 12 globular clusters and 67 field, by Butler $(A p J 200,68)$ with an image tube scanner. Metal abundances range from -0.39 for 6712 to -2.18 for $\mathrm{M} 92$.

\section{Population II Cepheids}

Demers and Harris $(A J$ 79) show that 25 Population II Cepheids in globular clusters, and 11 field variables define an instability strip 3 times wider than the strip for classical Cepheids. The variables form two luminosity groups. The position of the blue edge of the strip indicates the helium content of Population II Cepheids is $Y \approx 0.3$.

Böhm-Vitense $(A p J 188,571)$ calculates the effective temperature of Population II Cepheids from $B-V$ when gravity is known, for stars with different metal abundances. Later Böhm-Vitense, Szkody, Wallerstein and Iben $(A p J$ 194, 125) derived mean luminosities, effective temperatures and radii of 11 Population II Cepheids from 3 color photometry and some radial velocities. Masses of $0.55 \pm 0.05$ solar masses are found for all Population II Cepheids except Vars. 1 and 2 in $M 13$ which appear to be less massive and perhaps helium rich.

The position of the variables in the instability strip may be correlated with their amplitude, according to Caputo and Castellani $(A S S 19,423)$. The short period variables with $\log P<1$, which have the largest amplitude are the brightest and bluest, while longer period variables with $\log P>1$ show a reversed relation.

Bednarek (Doctoral Thesis, University of Toronto, 1975) from 200 linear, non-adiabatic models of Population II Cepheids finds a dichotomy in variables with $P>12$ days. Probably these are loop Cepheids while those with $P<8$ days are supra-horizontal branch stars.

\section{RV Tauri Variables}

Barnes and DuPuy $(A p J 200,364)$ confirm the period luminosity relation earlier determined by DuPuy for 4 stars in 4 globular clusters. Absolute magnitudes range from -4 for the 2 shortest periods to -3.0 for Var. 2 in NGC $6712,104.6$ days.

\section{Red Variables}

Kukarkin and Kireeva (Astr. Cirk. Izdav. Bjuro Astr. Soobsc. 808, 813) compute that red variable stars make good distance indicators for globular clusters with the following formula:

$$
\text { Mod } V_{\text {app }}=-0.80+1.03 B_{\text {max }} \pm 0.03-0.74(m / H) \pm 0.10
$$

and Kukarkin confirms the conclusion of Andrews, Feast, Lloyd Evans, Thackeray and Menzies that the period of their Mira stars is related to the metal content of the globular cluster. He suggests a search for Mira type in 9 clusters in which $m / \mathrm{H}$ exceeds -0.50 , nothing Var. $1=\mathrm{ZSge}$ in NGC 6838, with a periodicity of 360 days, and an amplitude increase sometimes of 3 mag.

In 47 Tucanae Lloyd Evans had near-infrared photometric measures for most of the red variables, and comments $(O b s 94,179)$ that the late $M$ giants in globular clusters are mostly 
variables of $\mathrm{L}, \mathrm{SR}$ or $\mathrm{M}$ type. Eggen $(A p J 195,661)$ shows that for large amplitude red variables the periods and median colors are well correlated.

\section{G. X-Ray Sources in Globular Clusters}

The report by Markert and Clark (Marsden, IAU Circ. 2735, 1974) of a variable X-ray source in NGC 1851 triggered much interest. A table by Clark ( $A p J$ 199, L143) summarizes the data on the 4 known sources, detected by Giacconi and collaborators, Clark, Markert, Li, Canizares and Neighbours. These are NGC 1851; MX 0513-40; NGC 6441, 3U 1746-37; NGC 6624, 3U 1820-30; NGC 7078, 3U 2131+11. Clark suggests that these may be binaries formed by capture from remnants of massive single stars which exploded, while Johnson (in press) suggests they may be stars recently condensed out of the ejecta of evolving red giants in these clusters.

\section{H. Variables in Clusters of External Galaxies}

\section{Large Magellanic Cloud}

Graham and Ruiz $(A J 79,363)$ have found 23 new variables in NGC 1835, a compact globular cluster near the central bar, rich in variables. They have determined periods and light curves for 18 RR Lyrae type, 11 of type $a b$ and 7 of type $c$, with the period distribution that of an Oosterhoff Type $I$ cluster. Demers $(A J 79,363)$ has determined light and color curves for 7 Cepheids in or near " 3 red globular clusters. Six are classical Cepheids, fall on the period luminosity relation for these stars, and are comparable to them in color. Demers concludes that probably they are not the Population II Cepheids such as are found in globular clusters of the galaxy.

\section{Small Magellanic Cloud}

Hodge and Wright $(A J \mathbf{8 0}, 510)$ give a table of data on 38 variables within or close to star clusters. About 21 are almost certain cluster members. There are 22 Cepheids, 3 eclipsing stars, 1 irregular, and 12 of unknown type.

\section{THEORY}

(N. Baker)

There have been several important recent conferences and symposia of which the subject of the theory of variable stars formed an important part. These include IAU Symp 59, 'Stellar Stability and Stellar Evolution', held in Canberra, 16-18 Aug., 1973; the 19th International Liège Astrophysical Colloquium, Problems in Stellar Hydrodynamics, Liège, 8-10 July, 1974; IAU Colloq. 29, 'Multiple Periodic Variable Stars', held in Budapest, 1-5 Sept., 1975; and an informal discussion on Cepheid Modeling among active workers in the field, at the Goddard Space Flight Center, Greenbelt, Md., 29-30 July, 1974. At these conferences, and elsewhere, several comprehensive reviews have appeared. The most complete is Pulsating Stars by J. P. Cox (Rep. Prog. Phys. 37, 563). Nonradial pulsations have received increasing attention, and the review of the theory by Ledoux (IAU Symp. 59) and the forthcoming review of theory and observations by J.P. Cox $(A R A A 14,1976)$ are especially recommended. Reviews of $\delta$ Sct and RRs type variables have been given by Baglin, Breger, Chevalier, Hauck, le Contel, Sareyan, and Valtier $(A A 23,221)$ and by Petersen (IAU Colloq. 29). The reader is referred to these reviews, and others presented at the conferences mentioned, for summaries and references to original work.

The review of greatest interest to members of this commission, however, is the introductory report to the 19th Liège Colloquium by J. P. Cox, entitled 'Stellar Oscillations, Stellar Stability, and Application to Variable Stars.' In section III of this paper, the author summarizes the recent applications of pulsation theory to the following classes of variable stars: Classical 
Cepheids, Double Mode Cepheids, RR Lyrae Variables, W Virginis and RV Tauri Variables, $\delta$ Sct Variables and Dwarf Cepheids, Long Period (Mira) Variables, Rapid Blue Variables, and $\beta$ Cephei Variables. The references are comprehensive and complete to mid-1974. It would be impossible to improve on this very compact summary, and in the present report no attempt is made to cover the same ground. We confine ourselves to bringing Cox's report up to date (references appearing there are for the most part excluded here) and to pointing to what seem to be the trends in the most recent work.

Theoretical work on Classical Cepheids continues. Recent papers by Karp (ApJ 199, 148; $200,354)$ include radiative transfer effects in the outer layers of nonlinear models, and good agreement with observed continuous spectra of cepheids was found. The author also discussed the period-luminosity relation, as did Iben and Tuggle ( $A p J 197,39)$. The topic of DoubleMode Cepheids of course received much attention at the Budapest Colloquium. The nonlinear mode interaction was investigated by Stellingwerf $(A p J 199,705)$. Petersen $(A A 34,309)$ found that artificial changes in opacity make rather little change in the period ratios of cepheid models. Although the apparent 'mass discrepancy' still exists for classical cepheid models, the masses of Population II Cepheids derived from observations by Böhm-Vitense, Szkody, Wallerstein, and Iben $(A p J 194,125)$ appear to agree with theoretical expectations.

There is still controversy regarding the masses and evolutionary status of $\delta$ Sct Variables and Dwarf Cepheids. Dziembowski (Act. Ast., in press) favors masses of the order $0.2 M_{\odot}$ for dwarf cepheids, and Dziembowski and Kozlowski (Act. Ast., 24, 245) computed linear nonadiabatic models for such stars. Some new linear nonadiabatic models with $1.5 M_{\odot}$ were reported by Pamyatnykh (Nauch Inf No. 29, 108) but nonlinear models, which would be very desirable, have not yet been reported.

The cause of the instability of the $\beta$ Cephei Stars remains unknown, although a number of theories have been advanced. It is possible that both radial and nonradial modes are involved, and possible resonances or beat phenomena have received attention, most recently from Deupree $(A p J 190,631 ; 194,393)$. Another type of resonance, in this case induced by tidal interactions with a companion, has been studied by Kato (PASJ 26, 341). Chiosi $(A A$ 37, 281) has suggested that nonradial gravity modes may become unstable during the overall contraction stage of massive stars, but an investigation by Osaki ( $P A S J$, in press) contradicts this.

Two papers on Dwarf Novae or the nova-like variables have appeared recently. Both Osaki (PASJ 26, 429) and Bath, Evans, Papaloizou, and Pringle $(M N 169,447)$ consider accretion models, in which gravitational energy is liberated by unstable mass transfer from the red star.

Two theoretical developments of some interest have been reported recently. The nature and ordering of the modes of nonradial pulsation in relatively highly condensed stars has been a problem for some years. Now Osaki (PASJ 27, 237) and, independently, Scuflaire $(A A 34,449$; $36,107)$ have suggested a natural ordering which gives insight into the behavior of these modes. It has been recognized, also, that ordinary nonadiabatic pulsation theory is not adequate for stars that are not in thermal equilibrium. The necessary theory has now been developed in a series of papers by Cox and collaborators $(A p J 182,855 ; 189,113 ; 191,439 ; 192, \mathrm{~L} 35 ; 194$, $663 ; 194,687 ; 195,175)$ and in an independent series by Demaret (Bull. Acad. Sci. Classe Sci. 60,$183 ; A S S 31,305 ; 33,189$ ). The applications to specific classes of variable stars of these two theoretical advances are not yet clear.

\section{ARCHIVES OF UNPUBLISHED OBSERVATIONS}

(W. S. Fitch)

The operation of the Commission 27 archives of photoelectric observations is described in Trans. IAU 15A, 313, 1973. The depositories presently contain 43 files, of which the first 22 were previously described. The new acquisitions are, briefly, files $I A U(27)$. RAS-

23. $145 U B V$ measures of RZ Cep by Epps and Sinclair.

24. 1530 differential $B$ measures of HL Tau-76 by Fitch.

25. 7932 differential $U B V$ measures of SX Aur, MN Cas, RW CrB, and V891 Cyg by Klawitter. 
26. 1111 differential $U B V$ measures of $21 \mathrm{Com}, 41 \mathrm{Tau}, \mathrm{HD} 140160$, and HD224801 by Blanco and Catalano.

27. $2954 B$ and $R$ measures of AR Lac by Kron, Gordon, Adams, and Smith.

28. $363 \lambda 8100 \AA$ measures of YY Gem by Kron and Gordon.

29. $U B V$ measures of CC Ser by Sarma and Parthasarathy.

30. UBV measures of HD140728, HD215038, and HD215441 by Blanco, Catalano, and Vaccari.

31. $1338 V$ measures of 1 Mon by Shobbrook and Stobie.

32. $4663 V$ measures of AC And by Szeidl.

33. $274 B V$ measures of SV Vol, GS Hya, VX Scl, and DH Vir by Penfold.

34. $950 u v b y$ measures of HD217061 by Lanning and James.

35. 4492 uvby measures of HDE226868=Cyg X -1 by Lanning.

36. $1812 U B V$ measures of RZ Cas by Chambliss.

37. $754 U B V$ measures of U peg by A. R. Hogg.

38. $4800 \mathrm{~V}$ measures of $\alpha \mathrm{Vir}, \lambda$ Sco, $\kappa$ Sco, $\beta \mathrm{CMa}, \xi \mathrm{CMa}$, and $15 \mathrm{CMa}$ by Shobbrook and Lomb.

39. $1280 U$ measures of TT Ari by Smak.

40. $2008 U B V$ measures of AR Lac by Chambliss.

41. 350 measures each of $U$ Oph in $50 \AA$ bands at $4750,4476,4230$, and $4026 \AA$, by Koch and Koegler.

42. $4855 b$ measures of $\delta$ Sct by Fitch.

43. $5574 \mathrm{~V}$ measures of $\mathrm{CC}$ And by Fitch.

\section{FLARE STARS}

(L. Rosino)

The study of flare stars has gained an increasing importance in these last years. Many papers dealing with flare stars in the solar neighbourhood as well as in clusters and associations have appeared: this review, for reasons of space, is reduced to the most significant contributions.

Excellent review papers on the problems of flare stars have been published recently by V. A. Ambartsumian and L. V. Mirzoyan (1975) and by W. E. Kunkel (1975); G. Godoli (1975) and R. E. Gershberg (1975) have summarized the aspects of the stellar activity of solar type, pointing out the many important common features between UV Ceti stars and solar phenomena. Other general contributions on the flare stars can be found in Part 1 of IAU Symp. 67 (D. Reidel, Dordrecht, 1975) held in Moscow in 1974, where appear, besides the papers already quoted, contributions of S. Cristaldi, M. Rodonò, W. E. Kunkel, D. Evans and others.

Systematic observations of flare stars in the solar neighbourhood (UV Cet stars) have been carried out at:

Catania. Photoelectric monitoring of flare stars has been continued actively, under the direction of Prof. G. Godoli, at Catania (Serra La Nave) mostly by S. Cristaldi and M. Rodonò. The following flare stars were systematically observed by the two authors:

$\begin{array}{ll}\text { YZ CMi }(I B 767) & \text { AD Leo }(I B 682,801) \\ \text { UV Cet }(I B 760,857) & \text { EQ Peg } \\ \text { BY Dra } & \text { V 371 Ori } \\ \text { EV Lac }(I B 759,802,836) & \text { V } 1216 \text { Sgr }(I B 721,835) \\ \text { PZ Mon } & \text { BD }+13^{\circ} 2618 \\ \text { G, 208-44/45 }(I A U C i r c 2805, A A, & \text { BD }+55^{\circ} 1863 \\ \quad \text { in press, 1975) } & \end{array}$

Rodonò (1974) has investigated with a high-speed photometer the Hyades flare star H 112411 , using the 208-cm telescope of McDonald, finding high frequency oscillations (13 $)$ superposed to normal flares. Light curves of these flare stars have been published by Cristaldi and Rodono 
(1973). Multi-peaked flares have been extensively studied. From a sample of about 500 flare events, almost $50 \%$ have been found to be double-peaked. Since about 60 of the flare stars in the solar neighbourhood are not single stars but binary or multiple systems, this has raised the question whether the double-peaked flares are the result of quasi-simultaneous flares in the two components. Rodono (preprint, 1975) has shown that this is really the case for the visual binary EQ Peg, although he has concluded that the proximity effect in general does not play an important triggering role.

McDonald Observatory. David Evans has sent an extended report on flare research carried out under his direction at the Department of Astronomy of Austin and McDonald Observatory by B. W. Bopp, T. J. Moffett, S. S. Vogt, R. M. Millan, C. H. Lacy and G. Ferland. The program, writes D. Evans, involved extensive studies by high speed photoelectric techniques of flare stars (Moffett, 1974a, b), simultaneous image tube spectroscopy of flares (Bopp, 1974), photometry and spectroscopy of stars showing starspots, statistical analysis of results, and scanner observations both of whole flare star spectra and of restricted emission regions (Evans, 1975). About 400 flares were observed by Moffett on 13 stars giving mean values for flare light: $B-V=+0.34 \pm 0.44, U-B=-0.88 \pm 0.31$. A general account of this cooperative effort is given by Moffett and Bopp (1976). Moffett found that most flares were combinations of two types of phenomena, a sharp spike of duration $10 \mathrm{~s}$ to few minutes and a slower flare of smaller amplitude and longer duration. The first has a continuum spectrum, the second an emission spectrum with Balmer, Ca II and sometimes He I lines in emission (Bopp and Moffett, 1973). He suggests that the spike flares represent discharge of energy into an atmospheric volume so small that it cannot be dissipated by ordinary recombination processes, but that this is possible for the slow flares.

The relationship between starspots and stellar flares has been closely investigated by Bopp and Evans (1973). Starspots have been observed on BY Dra, CC Eri and other stars. Bopp and Evans inferred high magnetic fields of the order of $30-50 \mathrm{kG}$ and found that the energy blocked by spots is of the same order as that released by flares. In spotted stars $V$ may vary as much as 0.3 mag. in a period of some days, while $B-V$ variations are small. Similar behaviour has been found for late $K$ and early $M$ dwarfs in the Pleiades.

Most flare stars are binary and both components flare. Rotation is rapid (from 5 to $44 \mathrm{~km}$ $\mathrm{s}^{-1}$ ) usually synchronous (excepting BY Dra).

Statistical analyses of Moffett's visual data (Lacy et al., 1975) yield the following results: There is no apparent correlation between times of flare occurrence. In particular, no firm evidence of precursor flares is yet found, in spite of special cases such as Wolf 424 (Moffett, 1973). Moreover no periodicity linked to rotation is found. There is no apparent correlation between flare energy and interval since the preceding flare. Flare energies in the $B, V$ and no filter observations are directly proportional to energies in the $U$ band. Intrinsically brighter flare stars have more energetic, but rarer flares.

Simultaneous optical and radio observations of flare stars were made by Moffett at McDonald and Spangler at Arecibo (Moffett, 1975; Spangler and Moffett, 1976). During simultaneous observations 62 optical flares were detected, with 28 radio enhancements within 10 min. of the optical events on 318 and/or $196 \mathrm{MHz}$. Extremely high level of polarization was found (Spangler et al., 1974) in a flare of AD Leo at $430 \mathrm{MHz}$ on 1974, April 1. The possibility of $\mathrm{X}$-ray emission from flare stars has also been considered (Crannell et al., 1974, 1975; Moffett, 1975).

Department of Geod. Astronomy of Thessaloniki. Mavridis, Asteriadis, Stavridis, Contadakis have continued at Thessaloniki the photoelectric patrol of selected flare stars: BD + $13^{\circ} 2618$, BD $+16^{\circ} 2708$, EV Lac, AD Leo, UV Cet $(I B \quad 712,809,812,1020)$, BY Dra, YZ CMi, BD $+55^{\circ} 1823$, Wolf 630, DO Cep. While observing UV Cet in 1972 one large flare was simultaneously observed at Thessaloniki in the optical region and at Jodrell Bank by Lovell with the 250-ft radio-telescope (1974).

A study of the variability of some UV Cet stars in the quiet state has been undertaken. Patrolling of flare stars has also been made at the Uttar Pradesh State Observatory, India, by Kapoor, Sanwal and Sinvhal. Five flares were observed in YZ CMi (IB 901, 998); 13 in AD Leo $(I B$ 901, 932) and 2 in EV Lac. 
Other surveys of flare stars have been made at the Tokyo Astronomical Observatory by Osawa, K., Ichimura, T. Okada, K. Okida, M. Vutani, H. Koyano (YZ CMi, IB 879) and by Ichimura, Y. Shimizu and Okida (1974) on the flare stars AD Leo and UV Cet.

A. N. Kulapova and N. I. Shakhovskaia (1973) obtained four spectrograms with dispersion $150 \AA \mathrm{mm}^{-1}$ during a flare of AD Leo. An asymmetry of emission line profiles was observed and a probable line shift of no more than $1 \AA$.

At the Crimean Astrophysical Observatory, Chugajnov (1974a, 1974b) has investigated the energy distribution in the spectrum of the excess radiation during a flare, with spectropolarimetric and $U B V$ measures. It is found that the continuous emission dominates. Chugajnov has also studied the photoelectric $B$ light curves of five flare stars (YZ CMi, UV Cet, EV Lac, AD Leo, V $1216 \mathrm{Sgr}$ ) finding that the mean duration of flares decreases with decreasing amplitude. Finally, Chugajnov (1974c) has made a photoelectric study on the light variability and the flare activity of red dwarf stars, in particular: FF And, Gliese 517, BY Dra and GT Peg, which are well-known variables of the BY Dra-type. Two new variables of this type: YZ CMi and BD- $10^{\circ} 4662$ have been identified. It was found that the flare activity in these stars is apparently independent of the periodical brightness variation. It is assumed that the light variability is due to axial rotation and presence of spots on the star surface.

\section{A. Flare Stars in Stellar Associations and Clusters}

\section{Pleiades}

G. Haro at Tonantzintla has continued the survey and study of flare stars in different associations. A complete catalogue of the flare stars in the Pleiades, with identification charts is in preparation. During winter 1972-73 Haro, Chavira and Gonzales (1973) have obtained 180 ultraviolet multiple exposure plates centered in Alcyone. In this material they have found 21 possible new flare stars and 102 outburst repetitions in previously known flare stars. Up to this time the brightest flare star in the Pleiades is $\mathrm{H} 111100$ with visual magnitude at minimum 12.16, spectral type K3Ve. Table 1 gives, according to Haro, Chavira and Gonzales, the brightest Pleiades flare stars and their characteristics at minimum:

Table 1

$\begin{array}{llllll}\text { Star } & V & B-V & U-B & m(U) & \text { Spectrum } \\ & & & & & \\ \text { H II } 97 & 12.50 & +1.08 & +0.79 & 0.8 & \\ \text { H II 324 } & 12.9 \mathrm{pg} & - & - & 1.4 & \\ \text { H II 1039 } & 12.97 \mathrm{pg} & +1.07 \mathrm{pg} & - & 0.7 \mathrm{pg} & \\ \text { H II 1100 } & 12.16 & +1.15 & +1.15 & 0.7 ; 1.3 & \mathrm{dK} 3 \mathrm{e} \\ \text { H II 1454 } & 12.78 & +1.16 & +0.94 & 1.0 & \mathrm{K3} \\ \text { H II 1553 } & 12.22 & +1.06 & +0.85 & 1.7 ; 1.5 & \\ \text { H II 1883 } & 12.60 & +1.06 & +0.71 & 2.0 ; 0.7 & \\ \text { H II 2034 } & 12.57 & +0.99 & +0.64 & 0.8 & \\ \text { H II 2244 } & 12.67 & +1.04 & +0.62 & 1.4 & \\ \text { H II 3197 } & 12.26 & +1.10 & +0.89 & 1.6 & \end{array}$

Ambartsumian et al. (1975) at Byurakan have given the results of photographic observations of flare stars (1972-73) in the Pleiades. 40 new flare stars and 66 repeated flares were found in the course of the survey. The number of observed flare stars in the region was 415 at the end of 1973. The total number of flare stars was estimated to be $\sim 1000$. Rosino and Pigatto, at Asiago, have also continued the systematic survey of flare stars in the Pleiades (1974). 150 flares were observed from the beginning of the survey. Other six flares were found on plates taken in the winter 1973-74 (unpubl.). The total number of new flare stars discovered at Asiago by the end of 1974 was 91 . It should be observed that a significant fraction of the flare 
stars found in Tonantzintla, Byurakan and Asiago in the Pleiades region, apparently are not members of the cluster.

Kholopov (1974) examining the spatial distribution of flare stars in the Pleiades concluded that weak and bright flare stars are concentrated towards the centre of the system.

Simultaneous optical (Palomar, Tonantzintla and Prairie Observatories) and radio (at the Vermillion River and Owens Valley Radio Observatories) observations of flare stars in the Pleiades were made from 1972 October 1 to 6 by Tomassian, Haro et al. (1974). Eleven optical flare-ups were detected. One large flare-up $\left(>8^{\mathrm{m}}\right.$ in $\left.U\right)$ was accompanied by a radio flare at $170 \mathrm{MHz}$. The ratio of optical to radio energy output of this flare was about $6 \times 10^{2}$.

Other seven flares in the Pleiades have been discovered by Szecsenyi-Nagy, with the Schmidt telescope of the Konkoly Observatory, Budapest (IB 898).

Flare stars searching has also been carried out in the following associations:

Orion. Fifteen stars showing rapid flare-up have been found with the Schmidt telescope of the Upsala Southern Station at Mount Stromlo by Roslund (1974) during $40 \mathrm{~h}$ of effective observation.

A. D. Andrews of the Armagh Observatory writes: work is continuing on $U B V R$ photometry of flare stars and $\mathrm{H} \alpha$ emission stars in the Orion nebula aggregate to establish their position in the colour-magnitude and two-colour diagrams. Tracks of NS Ori = T 157 within the diagrams, presumably typical of variables with very large ultraviolet excess, have been derived. Attention is drawn to a catalogue of astrometric and photometric $U B V R$ data for over 4000 stars in the Orion Nebula aggregate to $V=16$ at a single epoch. Computer-plotted $V$ charts and a statistical comparison of variables and non-variables in the Catalogue (Andrews, 1974) is under preparation.

Monoceros: NGC 2264. Systematic survey for the finding of flare stars in this region has been continued in the last two years by Rosino and it is still underway. The material is taken with the 92-67-cm Schmidt telescope of Asiago, with the method of multiple exposures.

Ophiuchus-Scorpio. Haro and Chavira (1974a) have found four new flare stars in an obscure area in this region where many emission objects are present (IB 926).

Cygnus near NGC 7000. Haro and Chavira have found seven flare stars in this region (1973). Some flare stars have also been detected by Rosino and Pigatto (1974, unpubl.). Systematic observations have been made since 1972 at the Byurakan Observatory by L. K. Erastova and M. K. Tsvetkov ( IB 909, 938, 1002), who found 38 new flare stars, some with repeated flares, in $560 \mathrm{~h}$ of observation. The total number of flare stars hitherto discovered in this region is 51 .

Coalsack. Five flare stars have been discovered in the Coalsack region by Hidayat and Kunkoro, Bosscha Observatory (1974) and three by McConnell and Dixon (1974). Their observations do not confirm the high rate of flaring previously reported.

Praesepe. Seventeen new flare stars have been found in this region by Jankovics (IB 839).

\section{REFERENCES}

Ambartsumian, V. A. et al.: 1975, ApJ 9, 4.

Ambartsumian, V. A. and Mirzoyan, L. V.: 1975, LAU Symp. 67, 3.

Andrews, A. D.: 1974 Bol. Tonantzintle 1, 101.

Bopp, B. W.: 1974, Dissertation, Univ. of Texas, Austin: $M N$ 168, 255.

Bopp, B. W. and Evans, D. S.: 1973, $M N$ 164, 343.

Bopp, B. W. and Moffett, T. J.: 1973, ApJ 185, 239.

Crannell, C. J., McClintock, J. E., and Moffett, T. J.: 1974, Nat 252, 659.

Crannell, C. J. et al.: 1975, Proc. 14th Int. Cosmic Ray Conf.

Chugainov, P. F.: 1974a, Izv. Krymsk. Astrofiz. Obs. 50, 93.

Chagainov, P. F.: 1974b, Izv. Krymsk. Astrofiz. Obs. 51, 110.

Chagainov, P. F.: 1974c, Izv. Krymsk. Astrofiz. Obs. 52, 3.

Cristaldi, S. and Rodonò, M: AASup. 10, 47.

Evans, D. S.: 1975, IAU Symp. 67,93.

Gershberg, R. E.: 1975, IAU Symp. 67, 47.

Godoli, G.: 1975, IAU Symp. 71. 
Hidajat, B. and Kunkoro, A: 1974, Publ. Bosscha Obs. Lembang 9.

Haro, G. and Chavira, E.: 1973, Bol. Tonantzintla 1, 17.

Haro, G. and Chariva, E.: 1974, Bol. Tonantzintla 1, 189.

Haro, G., Chariva, E., and Gonzales, G.: 1973, Bol. Tonantzintla 1, 1.

Ichimura, T., Smimizu, Y., and Okida, M.: 1974, Tokyo Astr. Bull. II Ser. 230.

Kholopov, P. N.: 1974, $A Z$ 51, 116.

Kulapova, A. N. and Shakhovskaja, N. I.: 1973, Izv. Krymsk. Astrofiz. Obs. 48, 31.

Kunkel, W. E.: 1975, IAU Symp. 67, 15.

Lacy, C. H., Moffett, T. J., and Evans, D. S.: 1975, ApJ Sup. (in print).

Lovell, B., Mavridis, L. N., and Contadakis, M.E.: 1974, Nat 250, 124.

MacConnell, D. J. and Dixon, M. S.: 1974, $A J 79,705$.

Moffett, T. J.: 1973, $M N 164,11$.

Moffett, T. J.: 1974a, ApJ Sup. 29, 1.

Moffett, T. J.: 1974b, Dissertation, Univ. of Texas, Austin.

Moffett, T. J.: 1975, IB 997.

Moffett, T. J. and Bopp, B. W.: 1976, ApJ Sup. (in press).

Rodonò, M.: 1974, $A A$ 32, 337.

Rosino, L. and Pigatto, L.: 1974, Mem. Soc. Astron. Ital. 44, 339.

Roslund, C.: 1974, Arkiv Astron. 5, 381.

Spangler, S. R. and Moffett, T. J.: 1976, ApJ (in press).

Spangler, S. R., Rankin, J. M., and Shawhan, S. D.: ApJ 194, L43.

Tovmassian, H. M. et al.: 1974, A strofiz. 10, 337.

\section{RR LYRAE VARIABLES \\ (L. Rosino)}

The determination of absolute magnitude of RR Lyrae variables is still an outstanding problem. Clube and Jones (1974) have critically discussed the method of determination of the absolute magnitude of RR Lyr variables in the solar vicinity from statistical parallaxes, finding the value $M_{y}=1.3 \pm 0.5$ more reliable than the value $M_{v}=0.6 \pm 0.8$ proposed by Heck (1972). The influence of possible errors in the technique for deriving statistical parallaxes was thoroughly discussed.

Studies on the light curves, periods and colours of RR Lyrae variables have been carried out in many Observatories. During the years 1970 to 1973 a selection of about 80 RR Lyr-stars south of $\delta=+15^{\circ}$ have been observed with the Walraven five-colours photometer at the Leiden Southern Station, mainly by van Genderen. Care was taken to have complete and continuous coverage of the light curves in order to study their form and small humps. J. Lub writes that the aim in this research was to find the physical parameters from the photometry and compare all of the information thus obtained with modern theoretical pulsation calculations.

D. H. P. Jones (1973) continued his studies of intermediate band photometry $(u, k, b, y$, $\beta$ systems) for 97 field RR Lyrae and some ultrashort-period variables. RR Lyrae variables in $\omega$ Cen and 47 Tuc were also considered. The colours are combined into indices which correlate with effective temperature, metal abundance, gravity and instellar reddening. From gravities and effective temperatures it is possible to obtain the mass-to-luminosity ratio and finally the masses, which are of the order of $0.5 M_{\odot}$ in good agreement with the theory.

A. W. Rodgers (1974) has proposed a new calibration of Preston's $\Delta \mathrm{S}$ parameter in terms of $\mathrm{Ca} / \mathrm{H}$, discussing its implications for the range of composition exhibited by field and cluster RR Lyrae variables. The peculiarity of some halo-type stars which appear metal-rich are pointed out.

L. Plaut (1973) with material taken at the European Southern Observatory has redetermined periods for all 104 RR Lyrae variables in Baade's field near NGC $6522\left(l=1^{\circ}, b=4^{\circ}\right)$. He has also derived the space distribution of these stars, finding the maximum density at a distance of $8.3 \mathrm{kpc}$. Plaut has also continued the Palomar-Groningen variables star survey, with $B$ and $V$ observations of $20 \mathrm{RR}$ Lyrae variables and the determination of photoelectric standard of reference.

J. A. Graham (1973) has dicussed the characteristics of the RR Lyrae stars in the Magellanic 
Clouds and NGC 1835, using also new material obtained with the Cerro Tololo telescopes. The periods and magnitudes of 29 RR Lyrae-ab variables in the globular clusters of the M.C. have been determined. All of them belong to Oosterhoff class $I$, with $\bar{P}_{a b}=0$ d 559 and $(\bar{B})=19.31$. The comparison between the magnitudes in LMC and SMC suggests that the latter has an apparent distance modulus $0 \mathrm{~m}^{\mathrm{m}} 4 \pm 0 \mathrm{~m} \cdot 2$ greater than the LMC. Graham and Ruiz (1974) have examined the field of NGC 1783 of LMC (which contains a strong Population I component). 72 Probable RR Lyrae variables have been found; for 51 of them periods have been determined. The most frequent period is again between $0 \mathrm{~d} 50$ and $0 \mathrm{~d} 55$ (Oosterhoff's class I). Other data are: $(\bar{B})=19.56 ;(\bar{V})=19.20$. The dispersion of the average blue magnitudes among LMC RR variables is very small, so indicating that these stars in the Cloud are concentrated in a disk system rather than spread on an extended halo system. On the field of SMC Graham finds: number of probable RR Lyr: 60; number of $\mathrm{RR}_{a b}$ with periods: 16. Most frequent period between 0955 and $0.60 ;(\vec{B}) \sim 20 ;(\bar{V}) \sim 19.6$. Assuming for the LMC a distance modulus 18.7 and for the SMC a modulus 19.2, the following mean values of $M_{v}$ are found: +0.5 (LMC); +0.4 (SMC).

Researches on the magnitudes, light curves and periods of RR Lyrae variables in globular clusters have been made by:

Belserene, E.: $\omega$ Cen (1973, LAU Colloq. 21, 105).

Cacciari, C. and Bologna: M 3 (1974, AA Sup 16, 63).

Dickens, R.J. and Flinn, R.: M 72 (1973, IAU Colloq. 21, 84).

Geyer, E. H.: $\omega$ Cen $(1973$, IAU Colloq. 21, 88).

Goranskij, V.P., Kukarkin, B. V., and Samus, N.N.: M 5, M 53, NGC 5053 (1973, IAU Colloq. 21, 101).

Goranskij, V. P.: M 53 (1974, Astr. Tsirk. 832).

Jones, D. H. P.: Cen, 47 Tuc (1973, LAU Colloq. 21, 84).

Menzies, J.: NGC $6723(1974, M N 168,177)$.

Pinto, G., and Rosino, L.: NGC 7006 (1973, Mem. Soc. Astron. Ital. 44, 255).

Rusev, R. M.: M $13(1973, \operatorname{Per} Z v 19,181)$.

Wesselink, A. J.: M 15, (1974, $A A$ 36, 163).

Wehlau, A. and Potts, N.: M 14 (1973, IAU Colloq. 21, 95).

Period and light curves of 35 RR Lyr variables, mostly of type $a b$, in the remote globular cluster NGC 2419 , have been derived by G. Pinto and L. Rosino (in print) by means of photographs taken at the 182-cm telescope of Asiago. The variables belong to Oosterhoff's class II,

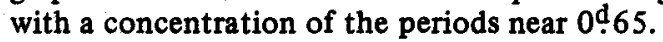

\section{A. Researches on Field RR Lyrae Variables}

G. A. Lange, B. N. Firmanuk, V. Bezdenezny at Odessa are regularly observing about 120 RR Lyrae stars, the ones with larger amplitude visually, those with small amplitudes photoelectrically.

J. Tremko at Skalnate Pleso Observatory has made three colours photoelectric observations of the two RR Lyr stars TT Lyn and W CVn. The first is a variable star of $R R_{a b}$ type with constant period and stable light curve. Blazko effect is not present. The second is $R R_{a b}$ type, with a hump in the ascending branch, most pronounced in $B$. The period is not constant, but it is decreasing slowly (Co. Skalnate Pleso 5, 159).

Georghe Chis et al., Astronomical Observatory Cluj, Rumania, have determined new elements and light curves for the RR Lyr variables: RU, SV, XX, WW Boo, DO Aqr, RZ Cep, XZ Cyg, WY Dra, XY Cyg $(1973,1974,1975 a)$. The period variation and Blazko effect in XZ Cyg has been studied by Vasile Pop (1975) while the Blazko effect in WY Dra has been observed and discussed by D. Chis, G. Chis and J. Mihoc (1975). The influence of rotation of RR Lyr stars in the radial velocity curve and adiabatic pulsations was examined by Lungu and Ureche (1975).

Photoelectric observations of X Ari, AR Per, RU Psc made by Mahra and Sinvhal, Uttar Pradesh State Observatory, India, show that the observed rates of period changes of these stars are in conformity with the rates predicted by theory and that these stars are evolving towards 
the red through the instability strip ( $I A U$ Colloq 29). The observed hump on the ascending branch of the RRa-type variables X Ari and AR Per is fully explained in terms of Abt's hypothesis of excess continuous emission resulting from a shock wave front propagating in the atmospheres of these stars.

\section{REFERENCES}

Chis, G., Chis, D., and Mihoc, 1.: 1973, Stud Cerc. Astr. 18, 139.

Chis, G., Chis, D., and Mihoc, I.: 1975, Studya Univ. Babes. Bolyai.

Clube, S. V. M. and Jones, D. H. P.: 1974, $A$ A 33, 154.

Graham, J. A.: 1973, IAU Colloq. 21, 120.

Heck, A.: 1972, $A A$ 21, 231.

Jones, D. H. P.: 1973, ApJ Sup 25, 225.

Rodgers, A. W.: 1974, $A p J$ 191, 433.

Plaut, L.: 1973, $A A$ 26, 317.

Pop, V.: $1975, I B 990$.

Todoran, I.: 1974, Stud Cerc. Astr. 19, 25; IB 915.

Ureche, V. and Lungu, N.: $1975, B A C 2$.

\section{REPORT ON BINARY X-RAY STARS FOR IAU \\ (H. Gursky)}

$\mathrm{X}$-ray astronomy changed qualitatively in 1970 with the dissemination of data from the satellite, Uhuru, which was the first one solely dedicated to cosmic X-ray observations. These data established the existence of the binary X-ray stars and the likelihood that most of the strong galactic $\mathrm{X}$-ray sources are of this kind. There are by now optically identified binary $\mathrm{X}$-ray stars and these are listed in Table 2 . There are clearly two kinds of stellar systems involved; one, as exemplified by Cyg X-1, 2U0900-40, 2U1700-37, Cen X-3 and SMC X-1 comprise a compact $X$-ray source and a young, massive star. The other class is not so distinctive except that the component masses are much closer to $\sim 1 M_{\odot}$. The results in this field through 1974 were discussed and reviewed at the 16th Solvay Conference (Giacconi, 1975; Bahcall, 1975). Based on these data a kind of standard model has emerged; one comprising a neutron star or black hole in a close binary system with a normal star (cf. Rees, 1975). Thus, the binary $\mathrm{X}$-ray stars form a class of objects similar to the cataclysmic variables except that a neutron star or black hole is substituted for the white dwarf. There are now four additional satellites yielding X-ray data (ANS, Ariel 5, SAS-3 and OSO 8). Two remarkable features which have emerged have to do with the transient $\mathrm{X}$-ray sources and the existence of $\mathrm{X}$-ray pulsations in

Table 2. Characteristics of Identified X-Ray Sources

\begin{tabular}{|c|c|c|c|c|}
\hline \multicolumn{2}{|l|}{ Name } & \multicolumn{2}{|r|}{ Ontical Companion } & Remarks \\
\hline $\begin{array}{r}3 U 1956+35 \\
1118-60 \\
0900-40 \\
1700-37 \\
0115-37\end{array}$ & $\begin{array}{l}\text { Cyg X-1 } \\
\text { Cen X-3 } \\
\text { Vela XR-1 } \\
\text { SMC X-1 }\end{array}$ & $\begin{array}{l}5.6 \text { days } \\
2.1 \text { days } \\
8.9 \text { days } \\
3.4 \text { days } \\
3.9 \text { days }\end{array}$ & $\begin{array}{l}9 \mathrm{mag} 0.97 \mathrm{Ib} \\
13 \mathrm{mag} \text { B0Ib-IIl } \\
6 \mathrm{mag} \text { B0. } 5 \mathrm{~Tb} \\
6 \mathrm{mag} 07 \mathrm{f} \\
13 \mathrm{mag} \mathrm{B0Ib}\end{array}$ & $\begin{array}{l}\text { 4.8-s X-ray pulse period } \\
284-\mathrm{s} \text { X-ray pulse period }\end{array}$ \\
\hline $\begin{array}{l}1617-15 \\
1653+35 \\
2030+40\end{array}$ & $\begin{array}{l}\text { Sco X-1 } \\
\text { Her X-1 } \\
\text { Cyg X-3 }\end{array}$ & $\begin{array}{l}0.8 \text { days } \\
1.7 \text { days } \\
0.2 \text { days }\end{array}$ & $\underset{-}{?} \stackrel{?}{15 \mathrm{~m} \text { late } \mathrm{A}}$ & $\begin{array}{l}\text { HZ-Her. 1.2-s pulse period } \\
\text { Heavily obscured, seen only } \\
\qquad \lambda>1 \mu\end{array}$ \\
\hline $2142+38$ & Cyg X-2 & $?$ & $14 \mathrm{mag} \mathrm{G}$ & \\
\hline
\end{tabular}


the several minute time range. Of the several transient X-ray sources discovered recently, one, in Centaurus, was found to have a period of $6.75 \mathrm{~min}$ (Ives et al., 1975) and the other in Taurus with a periodicity of $104 \mathrm{~s}$ (Eyles et al., 1975). Also, it is likely that one of the transient sources has been optically identified. A source in Monoceros, A0621-00, first reported by Elvis et al. (1975) has apparently been identified optically by Boley and Wolfson (1975) based on a position from the SAS-3 experiment (Matilsky, 1975). Eachus (1975) has found from studying material in the Harvard plate stacks that the optical object erupted once in the past and may be a recurring novae. Thus, this object may directly tie novae phenomena to the transient $X$-ray sources. However, there is as yet no direct evidence that the transient X-ray sources are binaries or that a white dwarf is involved; indeed Van Horn and Hansen (1974) have shown that it is possible to have novae-like explosions accompanying hydrogen accretion onto neutron stars.

The X-ray source 2U1900-37 was discovered to exhibit pulsations with a period of $283 \mathrm{~s}$ (Rappaport and McClintock, 1975) which exhibited a periodic Doppler shift with the known orbital period of the system. Thus, this object is a double line spectroscopic binary and it becomes possible, in principle, to determine a lower limit to the stellar masses. The X-ray emitter appears to be more massive than about $1.7 M_{\odot}$, which apparently excludes a white dwarf.

Within the past two years, three IAU Symposia have been held which had contributions relating to the binary $\mathrm{X}$-ray stars. These included IAU Symposium No. 67 held in Moscow (Sherwood and Plaut, 1975), Symposium No. 73 held in Cambridge, England (Mitton, 1975), and a Symposium sponsored by Commissions 42 and 44 held at the Goddard Space Flight Center (Kondo and Boldt, 1975).

\section{REFERENCES}

Bahcall, J. N. and Bahcall, N. A.: 1974, in 'Astrophysics and Gravitation', Proc. of the 16th Solvay Conf., Ed. Univ. Bruxelles, Belgique, 73.

Boley, F. and Wolfson, R.: 1975, IAU Circ. 2819, August 19.

Eachus, L. J.: 1975, IAU Circ. 2823, 27 August (reported by W. Liller).

Elvis, M. et al.: 1975, IAU Circ. 2814, August 8.

Eyles, C. G. et al.: 1975, IAU Circ. 2787 , June 2.

Giacconi, R.: 1974, in 'Astrophysics and Gravitation', Proc. of the 16th Solvay Conf., Ed. Univ. Bruxelles, Belgique, 27.

Ives, J. C., Sanford, P. W., and Burnell, S. J.: 1975, Nat. 254, 578.

Kondo, Y. and Boldt, E. (eds): 1975, 'Symposium on X-Ray Binaries', to be published by NASA.

Matilsky, T.: 1975, IAU Circ. 2819, August 19.

Mitton, S. (ed.): 1975, 'Close Binary Systems', Reidel, to be published.

Rees, M.: 1974, in 'Astrophysics and Gravitation', Proc. of the 16th Solvay Conf., Ed. Univ. Bruxelles, Belgique, 97.

Sherwood, V. and Plaut, L. (eds.): 1975, IAU Symp. 67.

Van Horn, H. M. and Hansen, C. J.: 1974, ApJ 191, 479.

\section{ABBREVIATIONS}

$\begin{array}{ll}A A A & =\text { Astronomy and Astrophysics Abstracts } \\ A A & =\text { Astronomy and Astrophysics } \\ \text { AA Sup } & =\text { Astronomy and Astrophysics Supplement } \\ \text { Act } A s t & =\text { Acta Astronomica } \\ \text { AJ } & =\text { Astronomical Journal } \\ A L & =\text { Astrophysical Letters } \\ A N & =\text { Astronomische Nachrichten } \\ \text { ApJ } & =\text { Astrophysical Journal } \\ \text { ApJ Sup } & =\text { Astrophysical Journal Supplement } \\ \text { ARAA } & =\text { Annual Review of Astronomy and Astrophysics } \\ \text { ASS } & =\text { Astrophysics and Space Science }\end{array}$




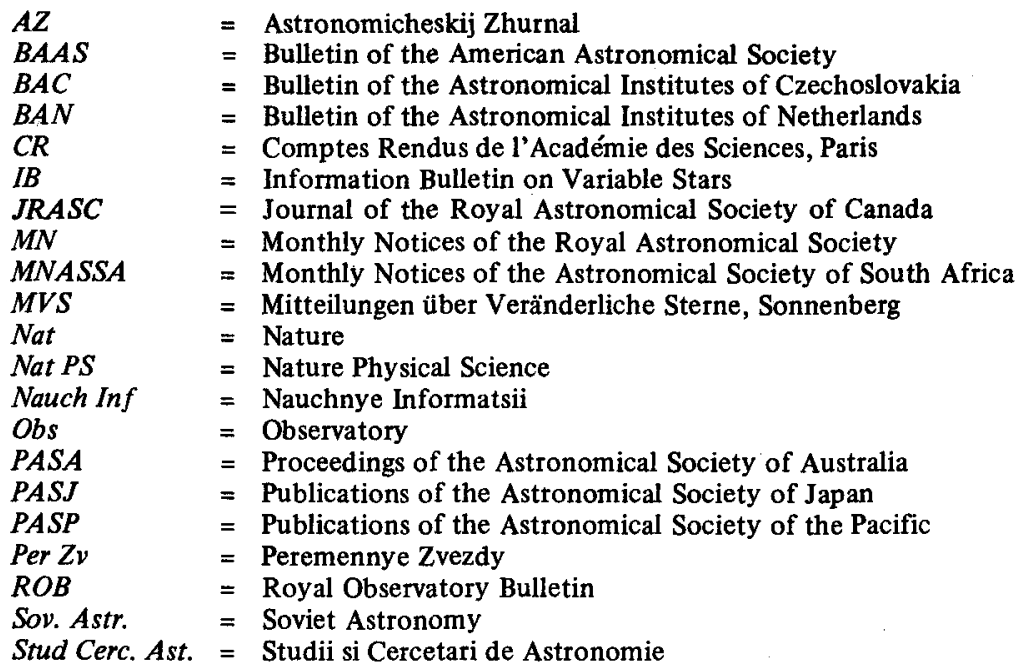

